\title{
HORIZONTAL VECTOR FIELDS AND SEIFERT FIBERINGS
}

\author{
ANDY HAMMERLINDL
}

\begin{abstract}
This paper gives a classification of the topology of vector fields which are nowhere tangent to the fibers of a Seifert fibering.
\end{abstract}

\section{INTRODUCTION}

This paper gives a classification of the topology of horizontal vector fields on Seifert fiber spaces. Here, horizontal means that the vector field is nowhere tangent to the Seifert fibering. This work was inspired in part by the classification of horizontal foliations on Seifert fiber spaces [Nai94]. Depending on the geometry of the 3-manifold, the condition of having a horizontal vector field can be either more restrictive or less restrictive than having a horizontal foliation. The key observation is that a horizontal vector field corresponds to a fiber-preserving map from the Seifert fiber space to the unit tangent bundle of the base orbifold.

We give definitions and review the theory for orbifolds and Seifert fiberings in sections 2 and 3 respectively, and develop a general theory for horizontal vector fields in section 4. In this introductory section, however, we first list out the possibilities based on the geometry of the base orbifold.

First and most interesting are Seifert fiber spaces where the base orbifold is a bad orbifold. These are lens spaces and each lens space $L(p, q)$ supports infinitely many distinct Seifert fiberings.

Theorem 1.1. Suppose $M$ is diffeomorphic to the lens space $L(p, q)$ with $p \geq 0$.

(1) If $p=1$ or $p=2$, then every Seifert fibering on $M$ has a horizontal vector field.

(2) If $p \geq 3$ and $q \equiv \pm 1 \bmod p$, then $M$ has infinitely many Seifert fiberings which support horizontal vector fields and infinitely many which do not.

(3) If $p \geq 8, p$ is divisible by 4 , and $q \equiv \frac{1}{2} p \pm 1 \bmod p$, then $M$ has exactly one Seifert fibering which supports a horizontal vector field and all others do not.

(4) For all other cases of $p$ and $q$, no Seifert fibering on $M$ has a horizontal vector field.

These cases are explained in detail in section 5. To prove theorem 1.1 we introduce the notion of a "marked lens space" which carries additional information on the orientation of the two solid tori which are glued together to construct the lens space. Under such a notion, $L(p, q)$ and $L(-p,-q)$ are distinct marked 
lens spaces even though they are diffeomorphic. Further, except for a few special cases, every Seifert fibering of $L(-p,-1)$ has a horizontal vector field while no Seifert fibering of $L(p, 1)$ has a horizontal vector field.

We next consider Seifert fiberings where the base orbifold is elliptic; that is, the orbifold is finitely covered by the 2-sphere. Some of these spaces, such as the 3-sphere, are also lens spaces and are handled by the previous theorem.

Theorem 1.2. Suppose $M$ is a Seifert fiber space with elliptic base orbifold and $M$ is not diffeomorphic to a lens space. Then $M$ has a horizontal vector field if and only if $M$, up to orientation, is the unit tangent bundle of the base orbifold.

Next consider Seifert fiber spaces over parabolic orbifolds. Such an orbifold is covered by the Euclidean plane and corresponds to a wallpaper group with only rotational symmetry.

Theorem 1.3. Suppose $M$ is a Seifert fiber space with parabolic base orbifold. Then $M$ has a horizontal vector field if and only if either

(1) $M$, up to orientation, is the unit tangent bundle of the base orbifold, or

(2) the base orbifold is a surface (either the 2-torus or the Klein bottle).

Section 7 explains both of the above cases in detail. Note that the two cases overlap when the base orbifold is a surface.

Finally, the most general case is for hyperbolic orbifolds.

Theorem 1.4. Suppose $M$ is a Seifert fiber space with hyperbolic base orbifold. Then the following are equivalent:

(1) $M$ has a horizontal vector field,

(2) M finitely covers the unit tangent bundle of the base orbifold,

(3) M supports an Anosov flow.

The equivalence (2) $\Leftrightarrow(3)$ in theorem 1.4 was proved by Barbot, extending a result of Ghys on circle bundles [Ghy84, Bar96]. The proof of (1) $\Leftrightarrow$ (2) is discussed in section 8 . Theorem 1.4 was part of the original motivation for exploring the properties of these vector fields. In joint work with Mario Shannon and Rafael Potrie, we analyze partially hyperbolic dynamical systems on Seifert fiber spaces [HPS18]. Most of that paper deals only with the case of dynamical systems defined on circle bundles. However, [HPS18, \$5] employs theorem 1.4 above to extend these results to the case of a general Seifert fiber space. See the cited paper for further details.

Section 9 studies the homotopy classes of horizontal vector fields, and section 10 looks at the case of Seifert fiberings on manifolds with boundary.

\section{ORBIFOLDS}

Intuitively, an orbifold is an object that locally resembles a quotient of $\mathbb{R}^{n}$ by a finite group. In this paper, we will only consider those orbifolds that appear as 
the base space of a Seifert fibering, and so we give a simpler definition restricted to this case.

Let $D^{2}=\{z \in \mathbb{C}:|z| \leq 1\}$ be the closed unit disk. For an integer $a \geq 1$, define $r_{a}$ : $D^{2} \rightarrow D^{2}, z \rightarrow e^{2 \pi i / a} z$. This is a rotation of order $a$. Define $D_{a}$ as the topological quotient space $D_{a}=D^{2} / r_{a}$. If $a>1$, then $D_{a}$ is homeomorphic to a disk, but is not a smooth surface. By a slight abuse of notation, we write $0 \in D_{a}$ for the image of $0 \in D^{2}$ under the quotient. If $U$ is an open subset of $D_{a}$, then $U$ is a smooth manifold if and only if $0 \notin U$. Let int $\left(D_{a}\right)$ denote the interior of $D_{a}$.

An orbifold $\Sigma$ is a closed topological surface equipped with an atlas of charts such that

(1) each chart is of the form $(\varphi, U, a)$ where $a \geq 1$ is an integer, $U \subset \Sigma$ is open, and $\varphi: U \rightarrow \operatorname{int}\left(D_{a}\right)$ is a homeomorphism;

(2) the charts cover $\Sigma$; and

(3) if $(\varphi, U, a)$ and $(\psi, V, \hat{a})$ are distinct charts in the atlas, then $\varphi(U \cap V)$ and $\psi(U \cap V)$ are smooth manifolds and $\psi \circ \varphi^{-1}: \varphi(U \cap V) \rightarrow \psi(U \cap V)$ is a diffeomorphism.

The final item above implies in particular that if $a>1$ then $\varphi^{-1}(0) \notin V$ and if $\hat{a}>1$ then $\psi^{-1}(0) \notin U$. We call $x \in \Sigma$ a cone point if the atlas has a chart $(\varphi, U, a)$ such that $\varphi(x)=0$ and $a>1$. Here, $a$ is the order of the cone point. In this version of the definition of an orbifold, each cone point is contained in exactly one chart. Since the orbifold is compact, it can have at most finitely many cone points.

In this paper, we refer to orbifolds using the orbifold notation of Thurston and Conley [Con92]. In this notation, the starting surface is a sphere and orbifolds are constructed from this by surgery. A positive integer $a$ in the notation corresponds to excising a disk and gluing in $D_{a}$ in its place. For example, the 237 orbifold is a sphere with cone points of orders 2,3 , and 7 added. We allow ones in the notation in order to make some results easier to state. For instance, consider the family of $p p$ orbifolds with $p \geq 1$. This is an infinite family of orbifolds that contains the 11 orbifold, i.e, the 2 -sphere, as a member of the family. The symbol " $\times$ " corresponds to replacing a disk with a cross cap, and so the $22 \times$ orbifold is the sphere with two cones points of order two and one cross-cap added. The symbol " $o$ " corresponds to adding a handle, and so the 23 oo orbifold is a genustwo surface with points of orders 2 and 3 added. We do not consider orbifolds with silvered edges or corner reflectors and so the "*" symbol will not occur in any of the notation here.

For an orbifold $\Sigma$, the underlying topological surface $\Sigma_{0}$ is just the orbifold itself treated as a topological surface and forgetting any of the additional information given by the charts $(\varphi, U, a)$.

For a smooth surface $\Sigma$ equipped with a Riemannian metric, one can define the unit tangent bundle $U T \Sigma$ consisting of all tangent vectors of length $\|\nu\|=1$. 
This is a 3-manifold $U T \Sigma$ with a natural circle fibering coming from the projection $U T \Sigma \rightarrow \Sigma$. We now extend this notion of a unit tangent bundle to the case of an orbifold.

First, consider a choice of Riemannian metric $g$ on the disk $D^{2}$ such that with respect to this metric the rotation $r_{a}: D^{2} \rightarrow D^{2}$ is an isometry. If $U$ is an open subset of $D_{a}$ with $0 \notin U$, then $g$ descends to a Riemannian metric on $U$. By a slight abuse of notation, we call such a $g$ a Riemannian metric on $D_{a}$. Define $T D_{a}$ as the quotient of $T D^{2}$ by the tangent map $T r_{a}: T D^{2} \rightarrow T D^{2}$. The tangent bundle $T D^{2}$ has the structure of a smooth 4-manifold with boundary, but if $a>1$, then the quotient $T D_{a}$ does not have such structure. Since $r_{a}$ is an isometry, its tangent map restricts to a diffeomorphism of the unit tangent bundle of $D^{2}$. This diffeomorphism and its iterates $\left.\operatorname{Tr}_{a}^{i}\right|_{U T D^{2}}$ for $1 \leq i<a$ have no fixed points. From this property, one can see that the quotient $U T D_{a}=U T D^{2} / T r_{a}$ is a smooth 3-manifold with boundary. In fact, it is a solid torus.

For an orbifold $\Sigma$, we define the tangent bundle using using equivalence classes of tangent vectors in charts. That is, consider the set $X$ of all tuples $(p,(\phi, U, a), v)$ where $p$ is a point in $\Sigma,(\phi, U, a)$ is a chart containing $p$, and $v$ a vector in $T D_{a}$ based at $\phi(p)$. The equivalence relation is defined by

$$
(p,(\phi, U, a), v) \sim(q,(\psi, V, \hat{a}), w)
$$

if and only if

$$
p=q \text { and } T\left(\psi \circ \phi^{-1}\right)(\nu)=w .
$$

The tangent bundle $T \Sigma$ of the orbifold is defined as the set of equivalence classes of $X$.

A Riemannian metric on an orbifold $\Sigma$ is a choice for every chart $(\phi, U, a)$ of Riemannian metric on $D_{a}$ such that every transition map $\psi \circ \phi^{-1}: \phi(U \cap V) \rightarrow$ $\psi(U \cap V)$ is an isometry. Suppose $\Sigma$ is an orbifold equipped with a Riemannian metric. As the transition maps are isometries, every element $v \in T \Sigma$ has a welldefined length, even those vectors based at cone points, and we may define the unit tangent bundle $U T \Sigma=\{v \in T \Sigma:\|v\|=1\}$.

A key observation is that even though $\Sigma$ is an orbifold, its unit tangent bundle is a smooth 3-dimensional manifold. To see this, note that $U T \Sigma$ is covered by open sets of the form $U T V$ where $V \subset \Sigma$ is given by a chart $(V, \phi, a)$ and $T \phi$ : $U T V \rightarrow U T D_{a}$ gives a smooth structure to $U T V$. Distinct choices of metric on $\Sigma$ will yield unit tangent bundles which are distinct as subsets of $T \Sigma$, but which are nonetheless diffeomorphic to each other. Because of this, in what follows we will at times discuss "the" unit tangent bundle of an orbifold without giving a specific choice of metric.

The unit tangent bundle of an orbifold is oriented if even the orbifold itself is not. This is because at a point $x \in \Sigma$, a choice of orientation of the tangent space $T_{x} \Sigma$ induces an orientation on the circle of unit tangent vectors. Regardless of the choice of orientation on $T_{x} \Sigma$, the resulting orientation on the 3-manifold is the same. 
One way to produce an orbifold is to quotient a surface by a discrete group of isometries. We call an orbifold $\Sigma$ a good orbifold if there is a Riemannian surface $S$ of constant curvature and a finite group of isometries $G$ acting on $S$ such that $\Sigma=S / G$ with the orbifold structure of $\Sigma$ coming from the smooth structure of $S$. We do not make a distinction between "good" and "very good" orbifolds as the notions are equivalent in the 2-dimensional setting. An orbifold is bad if it is not good. Proofs of the following two results are given in [Cho12, Chapter 5]. See also the discussion in [Sco83, §2].

Proposition 2.1. An orbifold $\Sigma$ is bad if and only if it is either

(1) a sphere with a single cone point of order $p>1$ added, or

(2) a sphere with two cone points of orders $p \neq q$ added.

For an orbifold $\Sigma$ with underlying topological surface $\Sigma_{0}$ and cone points of order $\alpha_{1}, \ldots, \alpha_{n}$, define the Euler characteristic of $\Sigma$ as

$$
\chi(\Sigma)=\chi\left(\Sigma_{0}\right)-\sum_{i}\left(1-1 / \alpha_{i}\right) .
$$

One way to think of this definition is that it is the formula $\chi(\Sigma)=V-E+F$ for vertices, edges, and faces, but with a cone point counting as a fraction $1 / \alpha_{i}$ of a vertex.

Proposition 2.2. If $\Sigma=S / G$ is a good orbifold, then $\chi(\Sigma)=\frac{1}{|G|} \chi(S)$.

We call a good orbifold $\Sigma$, elliptic if $\chi(\Sigma)>0$, parabolic if $\chi(\Sigma)=0$, and hyperbolic if $\chi(\Sigma)<0$. These cases correspond to the orbifold being covered by the 2-sphere, 2-torus, or a hyperbolic higher-genus surface respectively.

We may define vector fields on orbifolds analogously to vector fields on manifolds. If $\Sigma$ is an orbifold, a vector field on $\Sigma$ is a continuous function $v: \Sigma \rightarrow T \Sigma$ such that $\pi(\nu(x))=x$ for all $x \in \Sigma$ where $\pi$ is the canonical projection $\pi: T \Sigma \rightarrow \Sigma$.

Proposition 2.3. If $v$ is a vector field on an orbifold $\Sigma$, then $v$ is zero at every cone point on $\Sigma$. In particular, if $v$ is a non-zero vector field, then $\Sigma$ is a surface and is either the 2-torus or Klein bottle.

Proof. First, consider the case of a vector field defined on $D_{a}$ with $a>1$. Such a vector field lifts to a vector field $v: D^{2} \rightarrow T D^{2}$ which is invariant under the rotation $r_{a}$; that is, $\operatorname{Tr}_{a}(\nu(x))=v\left(r_{a}(x)\right)$ for all $x \in D^{2}$. If $x=r_{a}(x)$ is the center of the disk, this is only possible if the vector is zero at this point. This shows that a vector field must be zero at the cone point in $D_{a}$. Since orbifolds are locally modelled on $D_{a}$, any vector field on an orbifold must be zero at all cone points.

If an orbifold $\Sigma$ has a non-zero vector field, then $\Sigma$ must have no cone points and is a true surface. We are only considering the case of surfaces without boundary and the Poincaré-Hopf theorem implies that $\chi(\Sigma)=0$. 


\section{SEIFERT FIBERINGS}

We now discuss the definition and properties of Seifert fiberings.

Recall that $D^{2}$ is a closed unit disk and $r_{a}$ is a rotation of order $a$ on $D^{2}$. Let $S^{1}=\partial D^{2}$ and note that $r_{a}$ restricts to $S^{1}$. For coprime integers $a, b$ with $a \geq 1$, define a diffeomorphism $R_{a b}$ of the solid torus $D^{2} \times S^{1}$ by

$$
R_{a b}: D^{2} \times S^{1} \rightarrow D^{2} \times S^{1}, \quad(z, w) \mapsto\left(r_{a}^{b}(z), r_{a}(w)\right) .
$$

This generates a cyclic group of order $a$ acting freely on $D^{2} \times S^{1}$ and the quotient

$$
U_{a b}:=D^{2} \times S^{1} / R_{a b}
$$

is again a solid torus. Consider the projection $\Pi: D^{2} \times S^{1} \rightarrow D^{2}$ onto the first coordinate. The fibers of this projection are circles and this fibering is invariant under $R_{a b}$. Hence it defines a fibering of $U_{a b}$ by circles. The solid torus $U_{a b}$ along with this fibering by circles is called is called a standard fibered torus. The fibering may also be viewed directly as the fibers of a map from $U_{a b}$ to $D_{a}=$ $D / r_{a}$.

In the special case that $a=1, R_{a b}$ is the identity map and $U_{a b}=D^{2} \times S^{1}$ has a trivial fibering. We call this a trivial fibered torus.

Let $M$ be a closed 3-manifold along with a decomposition of $M$ into a disjoint union of circles. We say that $M$ is a Seifert fiber space if for every $x \in M$ there is a solid torus $U$ embedded in $M$ with $x$ in its interior such that $U$ is a union of circles and these circles give $U$ the structure of a standard fibered torus. That is, there are integers $a$ and $b$ and a diffeomorphism from $U_{a b}$ to $U$ which takes circles to circles.

Let $\Sigma$ be the topological space defined by quotienting $M$ along these circles. Then any standard fibered torus $U$ embedded in $M$ quotients down to a subset of $\Sigma$ with the same topological and smooth structure as the quotiented disk $D_{a}$. From this, one may show that the Seifert fibering on $M$ induces the structure of an orbifold on $\Sigma$. The projection $\pi: M \rightarrow \Sigma$ determines the fibering of circles on $M$, and so we will often refer to a Seifert fibering as given by a map $M \rightarrow \Sigma$ where $M$ is a closed 3-manifold and $\Sigma$ is an orbifold. If $x$ is a cone point in $\Sigma$, then the fiber $\pi^{-1}(x)$ is called an exceptional fiber. If $x$ is not a cone point, then $\pi^{-1}(x)$ is called a regular fiber.

We now outline the standard method of construction of a Seifert fiber space by gluing solid tori into a trivial circle bundle with boundary. This largely follows the exposition in [JN83]. Let $g \geq 0$ be an integer and let $\left(\alpha_{1}, \beta_{1}\right), \ldots,\left(\alpha_{n}, \beta_{n}\right)$ be such that for each $i, \alpha_{i}$ and $\beta_{i}$ are coprime integers and $\alpha_{i} \geq 1$. Let $F$ be a closed surface of genus $g$ and remove $n$ disjoint open disks from $F$ to produce $F_{0}=$ $F \backslash\left(D_{1}^{2} \cup \ldots \cup D_{n}^{2}\right)$. Let $M_{0}=F_{0} \times S^{1}$ be the trivial circle bundle over $F_{0}$ and write

$$
\partial M_{0}=\left(S_{1}^{1} \times S^{1}\right) \cup \ldots \cup\left(S_{n}^{1} \times S^{1}\right) .
$$


Define

$$
\begin{aligned}
R & =F_{0} \times\{1\}, \\
H_{i} & =\{1\} \times S^{1} \subset S_{i}^{1} \times S^{1}, \quad \text { and } \\
C_{i} & =R \cap\left(S_{i}^{1} \times S^{1}\right)=S_{i}^{1} \times\{1\} .
\end{aligned}
$$

Here, $H_{i}$ is a vertical fiber on the boundary of $M_{0}$ and $C_{i}$ is a horizontal circle around the excised disk. We assume $C_{i}$ has the same orientation as the boundary of the excised disk. This means that the boundary components of $R$ have the opposite orientation as the circles $C_{i}$.

Let $T_{i}=D^{2} \times S^{1}$ be a solid torus. It has a meridian $M_{i}=S^{1} \times\{1\} \subset \partial T_{i}$ which bounds a disk inside $T_{i}$ and a longitude $L_{i}=\{1\} \times S^{1} \subset \partial T_{i}$. Note that $M_{i}$ and $L_{i}$ form a basis for the first homology group of $\partial T_{i}$ and that $C_{i}$ and $H_{i}$ form a basis for the first homology group of $S_{i}^{1} \times S^{1} \subset \partial M_{0}$. At times, we consider rational linear combinations of elements of this homology, and so we use homology with rational coefficients throughout.

To glue $T_{i}$ into $M_{0}$, we choose a linear map identifying the two tori and such that $M_{i} \sim \alpha_{i} C_{i}+\beta_{i} H_{i}$ in homology. That is, we choose integers $\alpha_{i}^{\prime}$ and $\beta_{i}^{\prime}$ such that the matrix

$$
\left(\begin{array}{ll}
\alpha_{i} & \beta_{i} \\
\alpha_{i}^{\prime} & \beta_{i}^{\prime}
\end{array}\right)
$$

lies in $S L(2, \mathbb{Z})$ and use this as the gluing map. By a slight abuse of notation, we write

$$
\left(\begin{array}{c}
M_{i} \\
L_{i}
\end{array}\right)=\left(\begin{array}{cc}
\alpha_{i} & \beta_{i} \\
\alpha_{i}^{\prime} & \beta_{i}^{\prime}
\end{array}\right)\left(\begin{array}{c}
C_{i} \\
H_{i}
\end{array}\right)
$$

to concisely express how the circles are related in homology. Once $T_{i}$ is glued into the manifold, there is a unique way to extend the fibering to $T_{i}$ in such a way that $T_{i}$ is a standard fibered torus.

Note that the longitude of the solid torus is not well defined and we could replace $L_{i}$ with a curve homologous to $L_{i}+k M_{i}$ for any integer $k$. The gluing matrix is not unique and we could replace $\alpha_{i}^{\prime}$ and $\beta_{i}^{\prime}$ by $\alpha_{i}^{\prime}+k \alpha_{i}$ and $\beta_{i}^{\prime}+k \beta_{i}$. These, in fact, correspond to the same ambiguity and they have no impact on the topology of the resulting manifold.

After gluing in all of the tori, the result is a closed oriented 3-manifold. We denote this manifold equipped with this Seifert fibering as

$$
M\left(g ;\left(\alpha_{1}, \beta_{1}\right), \ldots,\left(\alpha_{n}, \beta_{n}\right)\right) .
$$

We now consider the case where $g<0$, which denotes a Seifert fibering over a non-orientable orbifold. Readers only interested in Seifert fiberings over oriented base orbifolds may safely skip over this paragraph. Let $g<0$ and let $F$ be a closed non-orientable surface of genus $|g|$. Let $F_{0}=F \backslash\left(D_{1}^{2} \cup \ldots \cup D_{n}^{2}\right)$. Let $G_{0}$ be the orientable double cover of $F_{0}$ and let $\tau: G_{0} \rightarrow G_{0}$ be an involution such that $F_{0}=G_{0} / \tau$. Define an involution $\phi$ on $G_{0} \times S^{1}$ by $\phi(g, z)=(\tau(g), \bar{z})$ where complex conjugation $z \mapsto \bar{z}$ is an involution of the circle. Then define a twisted 
circle bundle over $F_{0}$ by $M_{0}=\left(G_{0} \times S^{1}\right) / \phi$. Define $R$ as the image of $G_{0} \times\{1\}$ under this quotient. Note that while $M_{0}$ is orientable, the fibers are neither orientable nor transversely orientable on all of $M_{0}$. However, we can find a subset $M_{1}$ of $M_{0}$ which is a union of fibers, contains the boundary of $M_{0}$, and on which the fibers are both orientable and transversely orientable. This subset $M_{1}$ may be identified with $F_{1} \times S^{1}$ where $F_{1}$ is an orientable subset of $F_{0}$ and such that $R \cap M_{1}=F_{1} \times\{1\}$. Under this identification, the construction of the Seifert fibering by gluing proceeds exactly as before. Because of the existence of this subset $M_{1}$, we also freely assume in the proofs in the remainder of this section that the fibering is always orientable.

Two Seifert fiberings $M_{1} \rightarrow \Sigma_{1}$ and $M_{2} \rightarrow \Sigma_{2}$ are isomorphic if there is a diffeomorphism from $M_{1}$ to $M_{2}$ which takes fibers to fibers. Every Seifert fibering $M \rightarrow \Sigma$ of an oriented 3-manifold is isomorphic to one constructed by gluing in tori as above, and so we define the Seifert invariant of the fibering as

$$
\left(g ;\left(\alpha_{1}, \beta_{1}\right), \ldots,\left(\alpha_{n}, \beta_{n}\right)\right) \text {. }
$$

Two invariants define the same fibering if and only if one can be transformed into the other by changes of the following form:

(1) altering each $\beta_{i} / \alpha_{i}$ by an integer, but keeping $\sum \beta_{i} / \alpha_{i}$ fixed,

(2) re-ordering the pairs $\left(\alpha_{i}, \beta_{i}\right)$, and

(3) inserting or removing pairs of the form $(1,0)$.

For proofs of the above assertions, see [JN83, \$1] or [Hat07, \$2].

The Euler number of a fibering is defined as

$$
e(M \rightarrow \Sigma)=-\sum_{i=0}^{n} \beta_{i} / \alpha_{i}
$$

One can see that none of the above transformations to the Seifert invariant will affect the Euler number.

It is possible to extend the notation to define Seifert invariants for fiberings on non-orientable 3-manifolds. However, this complicates the notation and is not useful for the current paper, so we only consider invariants for oriented manifolds.

For most Seifert fiber spaces, the topology of the 3-manifold uniquely determines the fibering up to isomorphism. However, there are a few important exceptions, such as for lens spaces. We discuss this non-uniqueness in detail in section 5 ,

Recall that the unit tangent bundle $U T \Sigma$ of an orbifold $\Sigma$ is a closed 3-manifold. Further, the unit tangent bundle of a quotiented disk $D_{a}=D^{2} / r_{a}$ is a standard fibered torus where the fibers are given by the projection $T D_{a} \rightarrow D_{a}$. From this, one can see that the projection $U T \Sigma \rightarrow \Sigma$ gives $U T \Sigma$ a canonical Seifert fibering. 
Proposition 3.1. For an orbifold $\Sigma$ with underlying topological surface $\Sigma_{0}$ ofgenus $g$ and cone points of order $\alpha_{1}, \ldots, \alpha_{n}$, the unit tangent bundle has Seifert invariant

$$
\text { (g; } \left.\left(1, n-\chi\left(\Sigma_{0}\right)\right),\left(\alpha_{1},-1\right), \ldots,\left(\alpha_{n},-1\right)\right) .
$$

This fact is stated several times in the literature. See, for instance, EHN81, §5]. However, we know of no place that gives a complete proof including the case of bad orbifolds, and so we give a full proof here.

Proof. We will first consider the sphere and then certain quotients of the sphere before handling general surfaces and orbifolds.

For the unit tangent bundle of $S^{2}$, travelling once around a circle in the fibering corresponds to staying in one place on the globe, starting facing a certain direction, and then rotating once around counter clockwise (CCW). Consider a unit vector field on the 2-sphere defined everywhere except on small disks centered at the north and south poles. This unit vector field always points north and plays the role of $R$ in the construction of a Seifert fibering above.

Looking down on the north pole in a small chart, we may trivialize the unit tangent bundle in this chart. For instance, we may take a section of the unit tangent bundle to be a vector field which always points "up" inside this chart. If we consider a small disk around the north pole, then its unit tangent bundle is a solid torus and the meridian $M_{i}$ consists of vectors pointing "up" everywhere on the circle which bounds the disk. If we walk CCW around this circle once, then a north-pointing compass needle will make a complete CCW rotation. From this, one can see that $C_{i}$ is homologous to $M_{i}+H_{i}$. Similarly at the south pole, if we walk CCW once around the circle, then the compass needle will rotate once CCW. and so $C_{i} \sim M_{i}+H_{i}$ for this disk as well. Together, these show that the Seifert invariant of $U T S^{2}$ is $(0 ;(1,-1),(1,-1))$.

Now let $\alpha>1$ be an integer and the consider a rotation of order $\alpha$ of the sphere such that the quotient by this rotation is an orbifold $\Sigma$ and each of the north and south poles of the sphere quotients down to a cone point of order $\alpha$. This quotient defines a map $\pi: U T S^{2} \rightarrow U T \Sigma$ and one can verify that this a smooth covering map between manifolds. In what follows, we use uppercase letters $R, C_{i}, H_{i}, M_{i}, L_{i}$ for curves and surfaces in $U T S^{2}$ and lowercase letters $r, c_{i}, h_{i}, m_{i}, \ell_{i}$ for corresponding objects in $U T \Sigma$.

The north-pointing vector field is invariant under the rotation, and so it quotients down a vector field defined everywhere on the orbifold except for small disks about each of the cone point. The section $R$ defined in the previous case covers a section $r$ defined everywhere except on disks about the two cone points and the covering $R \rightarrow r$ is of degree $\alpha$.

Let $c_{i}$ denote a boundary circle of $r$, but with opposite orientation. It is $\alpha$ fold covered by $C_{i}$. Let $h_{i}$ denote a regular fiber which is the image of $H_{i}$ under the quotient. The north pole's meridional disk on $U T S^{2}$ maps down injectively to a meridional disk on $U T \Sigma$, and the boundary $m_{i}$ of this disk wraps $\alpha$ times 
around the cone point in $\Sigma$. Then

$$
\pi\left(C_{i}\right)=\alpha c_{i}, \quad \pi\left(H_{i}\right)=h_{i}, \quad \text { and } \pi\left(M_{i}\right)=m_{i}
$$

in homology. It follows from $C_{i} \sim H_{i}+M_{i}$ that $\alpha c_{i}$ is homologous to $h_{i}+m_{i}$. The exact same relations hold at the south pole. Hence, $m_{i} \sim \alpha c_{i}-h_{i}$ at both poles and the Seifert invariant for $U T \Sigma$ is $(0 ;(\alpha,-1),(\alpha,-1))$.

Now let $\Sigma$ be a surface of any genus. Put a vector field on $\Sigma$ which is nonzero everywhere except a finite set consisting of sinks, sources, and saddles. We can define small disks about each of the critical points and each disk yields a solid torus in the unit tangent bundle. As with the case of the sphere, each of the sinks and sources has a gluing with $M_{i} \sim C_{i}+H_{i}$. A "compass" following the vector field around a saddle will rotate once clockwise, and so one can show that a saddle corresponds to a gluing with $M_{i} \sim C_{i}-H_{i}$ Hence, the circle bundle has an Euler number which is the number of sinks and sources minus the number of saddles. The Poincaré-Hopf theorem tells us that this is exactly the Euler characteristic of the surface.

Finally, consider the general case of an orbifold $\Sigma$. Consider a vector field on the underlying surface $\Sigma_{0}$ which has finitely many sinks, sources, and saddles. Further assume that every cone point in $\Sigma$ corresponds to a point in $\Sigma_{0}$ which is a sink or source for the flow. Such a vector field may be produced, for instance, by considering a gradient flow where all of the cone points correspond to local minima or maxima. The previous case tells us that $U T \Sigma_{0}$ has a Seifert invariant which may be written as

$$
(g ;\left(1, n-\chi\left(\Sigma_{0}\right)\right), \underbrace{(1,-1), \ldots,(1,-1)}_{n \text { times }}) .
$$

Based on our above analysis of the 2-sphere and its quotient, one sees that the effect of removing a point in a surface and replacing it by a cone point of order $\alpha_{i}$ is to remove a pair $(1,-1)$ and replace it by $\left(\alpha_{i},-1\right)$ in the Seifert invariant. This then proves the desired result.

Corollary 3.2. The equality $(U T \Sigma \rightarrow \Sigma)=\chi(\Sigma)$ holds for any orbifold.

Proof. This follows directly from proposition 3.1 and the definitions of Euler number and Euler characteristic.

Suppose $M_{1} \rightarrow \Sigma$ and $M_{2} \rightarrow \Sigma$ are two Seifert fiberings over the same orbifold. We call a covering map $u: M_{1} \rightarrow M_{2}$ a fiberwise covering if it quotients down to the identity map on $\Sigma$. That is, the following diagram commutes:

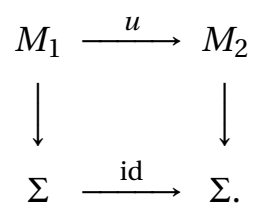


As described in [JN83, \$2], a Seifert fibering $M \rightarrow \Sigma$ may be viewed as the action of $S^{1}$ on the manifold where the orbits of the action are fibers of the fibering. One may also think of the fibers as being equipped with a metric such that every regular fiber has length exactly one. The metric varies continuously and so an exceptional fiber will then have a length of $1 / \alpha_{i}$. The $S^{1}$ action then corresponds to moving along the fibers at constant speed. A discrete subgroup of $S^{1}$ will be a cyclic group $\mathbb{Z} / d$ and we may consider the quotient of $M$ by $\mathbb{Z} / d$.

Proposition 3.3. Suppose $M$ is a Seifert fiber space with invariant

$$
\left(g ;\left(\alpha_{1}, \beta_{1}\right), \ldots,\left(\alpha_{n}, \beta_{n}\right)\right)
$$

and $d$ is a positive integer coprime to $\alpha_{i}$ for all $i$. Then the quotient $M /(\mathbb{Z} / d)$ is a Seifert fiber space with invariant

$$
\left(g ;\left(\alpha_{1}, d \beta_{1}\right), \ldots,\left(\alpha_{n}, d \beta_{n}\right)\right) .
$$

Moreover, any fiberwise covering of degree $d \geq 1$ between oriented Seifert fiber spaces is of this form.

Remark. A very similar result is stated as [JN83, Proposition 2.5], but without any distinction between the topological and smooth categories of manifolds. There, they allow the case where $d$ and $\alpha_{i}$ share a common factor. In this case, $\mathbb{Z} / d$ does not act freely on $M$. Further, the quotient destroys the smooth structure of $M$ and so $M /(\mathbb{Z} / d)$ is not a smooth manifold. As we are only concerned with smooth fiberwise coverings in the current paper, we do not allow such quotients.

To prove proposition 3.3, we first consider the effect of such quotienting on a standard fibered torus. For an integer $d>1$, define

$$
Q_{d}: D^{2} \times S^{1} \rightarrow D^{2} \times S^{1}, \quad(z, w) \mapsto\left(z, r_{d}(w)\right)
$$

and note that this map commutes with $R_{a b}$.

Lemma 3.4. Consider a standard fibered torus $U_{a b}=D^{2} \times S^{1} / R_{a b}$ with $a>1$ and the map $Q_{d}$ for some $d>1$.

(1) If a and $d$ are coprime, then the group $\left\langle R_{a b}, Q_{d}\right\rangle$ acts freely on $D^{2} \times S^{1}$ and the quotient is a standard fibered torus.

(2) If a and $d$ are not coprime, then the group $\left\langle R_{a b}, Q_{d}\right\rangle$ does not act freely on $D^{2} \times S^{1}$ and the quotient does not have the structure of a smooth manifold with boundary.

Proof. First, suppose $a$ and $d$ are coprime. Then, there are integers $n$ and $m$ such that $n d+m a=1$. Define $F$ as the composition $R_{a b}^{n} \circ Q_{d}^{m}$. Using

$$
n\left(\frac{b}{a}, \frac{1}{a}\right)+m\left(0, \frac{1}{d}\right)=\left(\frac{n b}{a}, \frac{1}{a d}\right) \in \mathbb{R}^{2},
$$

one can show that

$$
F(z, w)=\left(r_{a}^{n b}(z), r_{a d}(w)\right)=R_{\hat{a} \hat{b}}(z, w)
$$


where $\hat{a}=a d$ and $\hat{b}=n b d$. Further, $F^{a}=Q_{d}$ and $F^{d}=R_{a b}$, and so $\left\langle R_{a b}, Q_{d}\right\rangle$ is a cyclic group generated by $F$ and $D^{2} \times S^{1} / F$ is a standard fibered torus.

Now suppose $a$ and $d$ are not coprime. There is $0<n<a$ such that $n d$ is a multiple of $a$, and further there is $m$ such that $n d+m a=0$. In this case,

$$
R_{a b}^{n} \circ Q_{d}^{m}(z, w)=\left(r_{a}^{n}(z), w\right)
$$

and if $z=0$, then $(z, w)$ will be a fixed point. This shows that $\left\langle R_{a b}, Q_{d}\right\rangle$ does not act freely on the solid torus and that the quotient by this group does not have the structure of a smooth manifold with boundary.

Proof of proposition 3.3. Suppose $M$ is a Seifert fiber space as in the statement of the proposition. By lemma 3.4 and the fact that $d$ is coprime to $\alpha_{i}$ for all $i$, it follows that every standard fibered torus embedded in $M$ quotients down to a standard fibered torus in $M /(\mathbb{Z} / d)$. Hence, the latter is also a Seifert fiber space.

Let $R, C_{i}, H_{i}, M_{i}$, and $L_{i}$ be as in the construction of a Seifert fibering by gluing as described earlier and use $r, c_{i}, h_{i}, m_{i}, \ell_{i}$ for surfaces and curves in the covered manifold $M /(\mathbb{Z} / d)$. Let $\pi: M \rightarrow M /(\mathbb{Z} / d)$ be the quotient map. One can see that $\pi(R)=r, \pi\left(C_{i}\right)=c_{i}$, and $\pi\left(H_{i}\right)=d h_{i}$ in homology. For a solid torus neighbourhood of an exceptional fiber in $M$, a meridional disk maps injectively down to a meridional disk in $M /(\mathbb{Z} / d)$ and so $\pi\left(M_{i}\right)=m_{i}$. A longitude $\ell_{i}$ in the quotient is $d$-fold covered by a longitude of a solid torus in $M$. However, we do not know precisely which longitude covers $\ell_{i}$ and so the most we can say is that $\pi\left(L_{i}+k_{i} M_{i}\right)=d \ell_{i}$ for some integer $k_{i}$. Then

$$
\begin{aligned}
\pi\left(\begin{array}{c}
M_{i} \\
L_{i}
\end{array}\right) & =\left(\begin{array}{cc}
\alpha_{i} & \beta_{i} \\
\alpha_{i}^{\prime} & \beta_{i}^{\prime}
\end{array}\right) \pi\left(\begin{array}{c}
C_{i} \\
H_{i}
\end{array}\right) \Rightarrow \\
\left(\begin{array}{cc}
1 & 0 \\
-k_{i} & d
\end{array}\right)\left(\begin{array}{c}
m_{i} \\
\ell_{i}
\end{array}\right) & =\left(\begin{array}{cc}
\alpha_{i} & \beta_{i} \\
\alpha_{i}^{\prime} & \beta_{i}^{\prime}
\end{array}\right)\left(\begin{array}{cc}
1 & 0 \\
0 & d
\end{array}\right)\left(\begin{array}{c}
c_{i} \\
h_{i}
\end{array}\right) \Rightarrow \\
\left(\begin{array}{c}
m_{i} \\
\ell_{i}
\end{array}\right) & =\left(\begin{array}{cc}
\alpha_{i} & d \beta_{i} \\
\frac{1}{d}\left(\alpha_{i}^{\prime}+k_{i} \alpha_{i}\right) & \beta_{i}^{\prime}+k_{i} \beta_{i}
\end{array}\right)\left(\begin{array}{l}
c_{i} \\
h_{i}
\end{array}\right) .
\end{aligned}
$$

This formula would make sense whether or not the entries are integers as we are considering first homology with rational coefficients. However, since we already know $M /(\mathbb{Z} / d)$ is a well-defined Seifert fiber space, it must be that $\frac{1}{d}\left(\alpha_{i}^{\prime}+k_{i} \alpha_{i}\right)$ is an integer. This shows that the covered manifold has a Seifert invariant as stated in the proposition.

To prove the final statement, suppose $M_{1} \rightarrow M_{2}$ is a fiberwise covering of degree $d$. If we equip the fibers of $M_{2}$ with a metric such that every regular fiber has length exactly $1 / d$, this lifts by the covering to a metric on $M_{1}$ where every regular fiber has length exactly one. The quotient from $M_{1}$ to $M_{1} /(\mathbb{Z} / d)$ is then exactly the same as the quotient from $M_{1}$ to $M_{2}$.

We also wish to consider fiberwise coverings of negative degree. First, we consider the case of $d=-1$. 
Proposition 3.5. If $M$ has Seifert invariant $\left(g ;\left(\alpha_{1}, \beta_{1}\right), \ldots,\left(\alpha_{n}, \beta_{n}\right)\right)$, then $-M$ with the same fibering has invariant $\left(g ;\left(\alpha_{1},-\beta_{1}\right), \ldots,\left(\alpha_{n},-\beta_{n}\right)\right)$.

Proof. Let $M \rightarrow-M$ be the orientation-reversing identity map. To give $-M$ the opposite orientation as $M$, we may assume that the orientation along the fibers has been reversed, but the orientation transverse to the fibers remains the same. Then, keeping the same notation as in the previous proof, $\pi(R)=r, \pi\left(C_{i}\right)=c_{i}$, $\pi\left(H_{i}\right)=-h_{i}, \pi\left(M_{i}\right)=m_{i}$, and $\pi\left(L_{i}+k_{i} M_{i}\right)=-\ell_{i}$ for some integer $k_{i}$. One can then calculate the Seifert invariant of $-M$ much as in the previous proof.

Proposition 3.6. Suppose $M$ is a Seifert fiber space with invariant

$$
\left(g ;\left(\alpha_{1}, \beta_{1}\right), \ldots,\left(\alpha_{n}, \beta_{n}\right)\right)
$$

and $d$ is a non-zero integer coprime to $\alpha_{i}$ for all $i$. Then $M$ fiberwise covers a Seifert fiber space with invariant

$$
\left(g ;\left(\alpha_{1}, d \beta_{1}\right), \ldots,\left(\alpha_{n}, d \beta_{n}\right)\right) .
$$

Moreover, any fiberwise covering of degree d between oriented Seifert fiber spaces is of this form.

Proof. This follows from the previous two propositions.

Corollary 3.7. If $M_{1} \rightarrow \Sigma$ and $M_{2} \rightarrow \Sigma$ are Seifert fiberings and there is a fiberwise covering of degreed from $M_{1}$ to $M_{2}$, then $\cdot \cdot e\left(M_{1} \rightarrow \Sigma\right)=e\left(M_{2} \rightarrow \Sigma\right)$.

\section{General properties}

We now consider the general properties of horizontal vector fields on Seifert fiber spaces. Suppose $v$ is a vector field on a Seifert fiber space $M \rightarrow \Sigma$. That is, $v$ is a continuous map $v: M \rightarrow T M$ such that $v(x)$ lies in $T_{x} M$ for every $x$. We say that $v$ is horizontal if for all $x$, the vector $v(x)$ is not tangent to the fiber through $x$.

Proposition 4.1. A Seifert fibering $M \rightarrow \Sigma$ has a horizontal vector field if and only if there is a continuous map $u: M \rightarrow U T \Sigma$ such that

commutes.

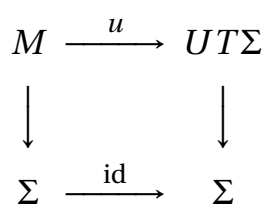

To prove this, we first show the equivalence inside a standard fibered torus. Recall the definitions of a standard fibered torus $U_{a b}=D^{2} \times S^{1} / R_{a b}$ and a quotiented disk $D_{a}=D^{2} / r_{a}$. Both $U_{a b}$ and the unit tangent bundles $U T D_{a}$ have fiberings given by canonical maps $\pi: U_{a b} \rightarrow D_{a}$ and $\pi_{a}: U T D_{a} \rightarrow D_{a}$.

Lemma 4.2. Any horizontal vector field on a standard fibered torus $U_{a b}$ induces a continuous fiber-preserving map from $U_{a b}$ to the unit tangent bundle of $D_{a}$. 
Proof. A vector field $v$ on $U_{a b}$ may be considered as a continuous function $v$ : $U_{a b} \rightarrow T U_{a b}$. Lifting the vector field to $D^{2} \times S^{1}$, we have a function $v_{1}: D^{2} \times S^{1} \rightarrow$ $T\left(D^{2} \times S^{1}\right)$ which satisfies

$$
v_{1} \circ R_{a b}=T R_{a b} \circ v_{1} .
$$

The lifted vector field is horizontal and for a point $y=(z, w) \in D^{2} \times S^{1}$ the vector $v_{1}(y)$ is not tangent to the fiber $\{z\} \times S^{1}$. In particular, if $\Pi: D^{2} \times S^{1} \rightarrow D^{2}$ is the projection onto $D^{2}$, then $T \Pi\left(v_{1}(y)\right) \in T_{z} D^{2}$ is non-zero. Define a map

$$
u_{2}: D^{2} \times S^{1} \rightarrow U T D^{2}, \quad y \mapsto \frac{T \Pi\left(\nu_{1}(y)\right)}{\left\|T \Pi\left(\nu_{1}(y)\right)\right\|} .
$$

One may show, using the definition of $R_{a b}$, that $u_{2}$ satisfies the symmetry relation $\operatorname{Tr}_{a}^{b} \circ u_{2}=u_{2} \circ T R_{a b}$. Hence, $u_{2}$ quotients to a map $u$ from $U_{a b}=D^{2} \times S^{1} / R_{a b}$ to $U T D_{a}=U T D^{2} / T r_{a}$. Moreover, as $u_{2}$ takes fibers to fibers, so does $u$.

Note that the above proof works for any choice of Riemannian metric on $D_{a}$.

Lemma 4.3. Let $U_{a b}$ be a standard fibered torus, $P$ a plane field on $U_{a b}$ transverse to the fibering, and $u: U_{a b} \rightarrow U T D_{a}$ a continuous function for which

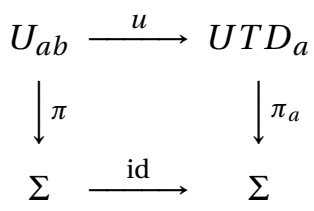

commutes. Then there exists a unique horizontal vector field $v: U_{a b} \rightarrow T U_{a b}$ such that for all $x \in U_{a b}, v(x) \in P(x)$ and $T \pi(\nu(x))=u(x)$.

Proof. Each of $U_{a b}$ and $U T D_{a}$ is a solid torus with an infinite cyclic fundamental group. Since both covering maps $D^{2} \times S^{1} \rightarrow U_{a b}$ and $U T D^{2} \rightarrow U T D_{a}$ are of the same degree $a$, one may verify that the lifting criterion [Hat02, Proposition 1.33] applies here and $u: U_{a b} \rightarrow U T D_{a}$ lifts to a map $u_{1}: D^{2} \times S^{1} \rightarrow U T D^{2}$. Moreover, we may choose the lift in such a way that

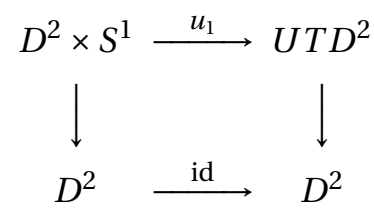

commutes. To see this, first choose a lift so that the above diagram commutes for at least one point $y \in D^{2} \times S^{1}$, and then use that $D^{2} \times S^{1}$ is connected to show that it commutes for all points. The lifted map satisfies $u_{1} \circ R_{a b}=\operatorname{Tr}_{a}^{n} \circ u_{1}$ for some integer $n$, and the above commutative diagram shows that $n \equiv b \bmod a$. Without loss of generality, assume $n=b$.

The transverse plane field $P$ on $U_{a b}$ lifts to a transverse plane field $P_{1}$ on $D^{2} \times S^{1}$ which is invariant under $T R_{a b}$. Let $\Pi: D^{2} \times S^{1} \rightarrow D^{2}$ be the canonical projection. By transversality, for each point $y=(z, w) \in D^{2} \times S^{1}$ the tangent map

$$
T_{y} \Pi: T_{y}\left(D^{2} \times S^{1}\right) \rightarrow T_{z} D^{2}
$$


restricts to an isomorphism from the 2-dimensional subspace $P(y)$ onto $T_{z} D^{2}$. Define $u_{2}(y)$ as the unique vector in $P(y)$ which satisfies $T \Pi\left(v_{2}(y)\right)=u_{1}(y) \in$ $T_{z} D^{2}$. Doing this at all points in $D^{2} \times S^{1}$ defines a vector field $v_{2}: D^{2} \times S^{1} \rightarrow$ $T\left(D^{2} \times S^{1}\right)$.

Using $P\left(R_{a b}(y)\right)=T R_{a b}(P(y))$ and $u_{1}\left(R_{a b}(y)\right)=\operatorname{Tr}_{a}^{b} u_{1}(y)$, one can see that $\left.v_{2}\left(R_{a b}(y)\right)=T R_{a b} v_{2}(y)\right)$. Hence, $u_{2}$ quotients down to a vector field $u: U_{a b} \rightarrow$ $T U_{a b}$ and this is the desired vector field. Since $u_{2}$ is uniquely determined, so is $u$.

Proof of proposition 4.1. Suppose $v: M \rightarrow T M$ is a horizontal vector field. Define a function

$$
u: M \rightarrow U T \Sigma, \quad x \mapsto \frac{T \pi(\nu(x))}{\|T \pi(v(x))\|}
$$

where $\pi: M \rightarrow \Sigma$ defines the fibering. As $v$ is horizontal, $u$ is well defined. Any point $x \in M$ lies inside of a standard fibered torus, where the definition of $u$ agrees with the map given in lemma 4.2 . Hence, $u$ is continuous and is the desired map.

For the converse direction, suppose $u: M \rightarrow U T \Sigma$ is a map as in the statement of the proposition. Choose a plane field $P$ transverse to the fibering. Such a plane field always exists; for instance, one can equip $M$ with a Riemannian metric and consider the planes which are orthogonal to the fibers. Then define $v: M \rightarrow T M$ as the unique vector field tangent to $P$ which satisfies $T \pi(\nu(x))=$ $u(x)$ for all $x \in M$. By lemma 4.3, such a $v$ exists and is continuous. Moreover, $v$ is horizontal by construction.

Fiber-preserving maps must be of a specific form.

Proposition 4.4. Suppose $M_{1}$ and $M_{2}$ are Seifert fiber spaces over the same base orbifold $\Sigma$ and $u: M_{1} \rightarrow M_{2}$ is a fiber-preserving map such that

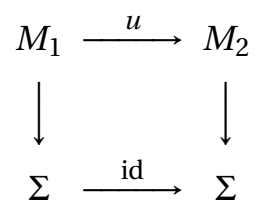

commutes. Then either

(1) $u$ is homotopic to a composition of the form $M_{1} \rightarrow \Sigma \rightarrow M_{2}$, or

(2) $u$ is homotopic to a fiberwise covering $M_{1} \rightarrow M_{2}$.

Remark. In the first case, the map $M_{1} \rightarrow \Sigma$ is the projection defining the fibering and the second map $\Sigma \rightarrow M_{2}$ is a continuous section for the fibering $M_{2} \rightarrow \Sigma$. In either case, the homotopy is along the fibers of $M_{2}$, as may be seen from the proof below.

Proof. First assume that $M_{1}$ and $M_{2}$ and their fibers are orientable. Because $u$ projects down to the identity map on $\Sigma$, the degree of $u$ is equal to the degree of the restriction of $u$ to any fiber of $M_{1}$. Further, since the fiberings of $M_{1}$ and $M_{2}$ 
are smooth, we may perturb the continuous map $u$ slightly to produce a smooth function which also projects to the identity on $\Sigma$. This smooth perturbation is homotopic to $u$, and so without loss of generality, we assume $u$ itself is smooth.

Equip each of $M_{1}$ and $M_{2}$ with a metric so that every regular fiber has length exactly one. The exceptional fibers will then have lengths $1 / \alpha_{i}$ for integers $\alpha_{i}>$ 1. Consider a point $x \in M_{1}$ and let $L_{1}$ be the fiber through $x$ and $L_{2}$ the fiber through $u(x)$. Define length-preserving covering maps $\pi_{1}: \mathbb{R} \rightarrow L_{1}$ and $\pi_{2}: \mathbb{R} \rightarrow$ $L_{2}$ and choose a lift $\tilde{u}: \mathbb{R} \rightarrow \mathbb{R}$ such that $\pi_{2} \circ \tilde{u}=u \circ \pi_{1}$. Define $\hat{u}: \mathbb{R} \rightarrow \mathbb{R}$ by

$$
\hat{u}(t)=\int_{t-\frac{1}{2}}^{t+\frac{1}{2}} \tilde{u}(s) d s \quad \text { so that } \quad \frac{d \hat{u}}{d t}=\tilde{u}\left(t+\frac{1}{2}\right)-\tilde{u}\left(t-\frac{1}{2}\right)=\operatorname{deg}(u) .
$$

There is then a unique function $h: L_{1} \rightarrow L_{2}$ such that $h \circ \pi_{1}=\pi_{2} \circ \hat{u}$ and $h$ is independent of the choice of lift $\tilde{u}$. When defined fiber by fiber on all of $M_{1}$, this gives a smooth map $h: M_{1} \rightarrow M_{2}$. By shrinking the interval [ $\left.t-1 / 2, t+1 / 2\right]$ down to a point, one can show that $h$ is homotopic to $u$ and that the homotopy is through fiber-preserving maps.

If $\operatorname{deg} u=0$, then $h$ is constant on every fiber of $M_{1}$, and so it factors as a composition $M_{1} \rightarrow \Sigma \rightarrow M_{2}$. If $\operatorname{deg} u \neq 0$, then $h$ is a covering map on each fiber. Since $h$ projects to the identity on $\Sigma$, one can see that it is a global covering map $M_{1} \rightarrow M_{2}$.

If any of $M_{1}, M_{2}$, or the fiberings is not orientable, the steps above still work. However, the degree of $u$ is only defined up to a sign. The interval $[t-1 / 2, t+1 / 2]$ was used above in order to avoid having to choose an orientation on a fiber.

Because of proposition 4.1, we primarily consider proposition 4.4 in the case where $M_{2}$ is a unit tangent bundle. When is the case $M \rightarrow \Sigma \rightarrow U T \Sigma$ possible? This requires a horizontal section $\Sigma \rightarrow U T \Sigma$; that is, a vector field on $\Sigma$ which by proposition 2.3 is possible only if $\Sigma$ is the 2-torus or Klein bottle. This situation is discussed in further detail in section 7 , Outside of this case, we have the following equivalences.

Theorem 4.5. Let $M \rightarrow \Sigma$ be a Seifert fibering over a base orbifold $\Sigma$ which is neither the 2-torus nor the Klein bottle. Then the following are equivalent:

(1) $M$ has a horizontal vector field;

(2) there is a continuous map $u: M \rightarrow U T \Sigma$ such that

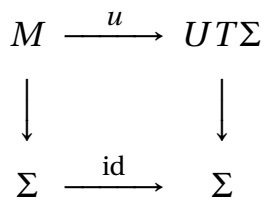

commutes;

(3) there is a fiberwise covering from $M$ to the unit tangent bundle UT $\Sigma$;

(4) $M$ is orientable, the fibering has Seifert invariant

$$
\left(g ;\left(\alpha_{1}, \beta_{1}\right), \ldots,\left(\alpha_{k}, \beta_{k}\right)\right)
$$


and there is a non-zero integerd such that $\cdot e(M \rightarrow \Sigma)=\chi(\Sigma)$ and

$$
d \beta_{i} / \alpha_{i} \equiv-1 / \alpha_{i} \quad \bmod \mathbb{Z}
$$

for all $i=1, \ldots, k$.

Proof. The equivalence (1) $\Leftrightarrow$ (2) is given by proposition 4.1 and (3) $\Leftrightarrow$ (4) is a combination of propositions 3.1 and 3.6. Note that as $U T \Sigma$ is oriented, any manifold $M$ covering $U T \Sigma$ is orientable. The direction $(2) \Leftarrow(3)$ follows immediately from the definition of a fiberwise covering. The only place we use that $\Sigma$ is not the 2-torus or Klein bottle is to invoke proposition 2.3 to ensure we are in the second case of proposition 4.4. This gives (2) $\Rightarrow$ (3).

The integer $d$ in item (4) above has topological significance in the other three items as well. In (2) and (3), $d$ is the degree of the map $u: M \rightarrow U T \Sigma$. In (1), $d$ is the number of turns that the vector field makes while going around a regular fiber.

In the next four sections, we consider the consequences of theorem 4.5 for the cases of bad, elliptic, parabolic, and hyperbolic base orbifolds respectively.

\section{LENS SPACES}

We now consider Seifert fiberings on lens spaces. To aid in the study, we introduce in this paper the notion of a "marked" lens space.

For a solid torus, $D^{2} \times S^{1}$, the core of a solid torus is the curve $\{0\} \times S^{1}$ where 0 is the center of the disk $D^{2}$. A lens space is a closed oriented 3-manifold $L$ constructed by gluing together together two solid tori $U_{1}$ and $U_{2}$ by a linear map $A: \mathbb{T}^{2} \rightarrow \mathbb{T}^{2}$ which identifies the two boundaries. A marked lens space is a lens space along with a choice of orientation for each of the cores of the two solid tori. A fibered marked lens space is a marked lens space equipped with a Seifert fibering such that each of $U_{1}$ and $U_{2}$ are standard fibered tori.

Later in this section, we show that there are two families of Seifert fiberings on lens spaces. One of these is the fibered marked lens spaces. The other is produced by taking a single standard fibered torus and, by identifying points on the boundary, closing it up into a closed 3-manifold. We first fully treat the fibered marked lens spaces before handling this second case.

5.1. Fibered marked lens spaces. Now consider a marked lens space $L$. For the boundaries of the two solid tori, the meridians $M_{i}$ are well defined (at least up to homology) as they bound solid disks. The longitudes, however, are not well defined. If $L_{i}$ is a longitude on the boundary of $U_{i}$, then for any integer $k_{i}$, there is a diffeomorphism from $U_{i}$ to itself which maps $L_{i}$ to a curve homologous to $L_{i}+k_{i} M_{i}$. As we are assuming the core of $U_{i}$ has a specified orientation, we only consider longitudes with a compatible orientation. 
Viewing the gluing map $A$ as two-by-two matrix, we have

$$
\left(\begin{array}{c}
M_{1} \\
L_{1}
\end{array}\right)=A\left(\begin{array}{c}
M_{2} \\
L_{2}
\end{array}\right)
$$

in homology. In order for the lens space $L$ to be an oriented 3-manifold the gluing map must reverse the orientation of the boundary, and so this matrix has a determinant equal to -1 . In this section, we write this gluing matrix as

$$
A=\left(\begin{array}{cc}
-q_{2} & p \\
* & q_{1}
\end{array}\right)
$$

with a $*$ for the lower-left entry. We omit this entry as it is uniquely determined by $\operatorname{det}(A)=-1$ and the other three entries, and whereas the other entries have topological significance, this lower-left entry is usually given by a relatively complicated formula with no particular significance.

The matrix depends on the choice of longitudes $L_{i}+k_{i} M_{i}$ and a different choice of longitudes would yield a matrix

$$
\left(\begin{array}{cc}
1 & 0 \\
k_{1} & 1
\end{array}\right)\left(\begin{array}{cc}
-q_{2} & p \\
* & q_{1}
\end{array}\right)\left(\begin{array}{cc}
1 & 0 \\
k_{2} & 1
\end{array}\right)^{-1}=\left(\begin{array}{cc}
-q_{2}-p k_{2} & p \\
* & q_{1}+p k_{1}
\end{array}\right) .
$$

This shows that the integer $p$ is well defined and that $q_{1}$ and $q_{2}$ are well defined modulo $p$. If we relabelled $U_{1}$ and $U_{2}$ as $U_{2}$ and $U_{1}$ respectively, then the gluing map would be replaced by its inverse, and the matrix would then be

$$
\left(\begin{array}{cc}
-q_{2} & p \\
* & q_{1}
\end{array}\right)^{-1}=\left(\begin{array}{cc}
-q_{1} & p \\
* & q_{2}
\end{array}\right)
$$

Note also that the condition $\operatorname{det}(A)=-1$ implies that $q_{1} q_{2} \equiv-1 \bmod p$.

For coprime integers $p$ and $q$, define $L(p, q)$ as the marked lens space constructed by gluing together two solid tori by a gluing map with a matrix of the form

$$
\left(\begin{array}{cc}
-q_{2} & p \\
* & q_{1}
\end{array}\right)
$$

where $q_{1}=q$ and the first column is chosen so the determinant is -1 . As the choice of the first column of the matrix corresponds only to a choice of longitude on $U_{1}$, the marked lens space $L(p, q)$ is well defined. Further, for any integer $k$, $L(p, q)$ and $L(p, q+p k)$ are the same marked lens space. If $q_{1} q_{2} \equiv-1 \bmod p$. then $L\left(p, q_{1}\right)$ and $L\left(p, q_{2}\right)$ are the same marked lens spaces, differing only by the choice of labelling of the two solid tori as $U_{1}$ and $U_{2}$.

Now suppose we take a marked lens space and produce a new marked lens space by reversing the orientation of the core of exactly one of the two solid tori, say $U_{2}$. This reverses the orientation of the longitude $L_{2}$ and, in order to keep the original orientation of the 3-manifold unchanged, we must reverse the orientation of the meridian $M_{2}$ as well. With respect to these new orientations, the 
gluing matrix is now given by

$$
\left(\begin{array}{cc}
-q_{2} & p \\
* & q_{1}
\end{array}\right)\left(\begin{array}{cc}
-1 & 0 \\
0 & -1
\end{array}\right)=\left(\begin{array}{cc}
q_{2} & -p \\
* & -q_{1}
\end{array}\right)
$$

This leads to the following key observation:

$L(p, q)$ and $L(-p,-q)$ are diffeomorphic as oriented 3-manifolds, but they are not the same marked lens space.

We can reverse the orientation of a lens space, say by leaving the orientations of both cores unchanged, but reversing the orientations of both meridians. The new gluing matrix is

$$
\left(\begin{array}{cc}
1 & 0 \\
0 & -1
\end{array}\right)\left(\begin{array}{cc}
-q_{2} & p \\
* & q_{1}
\end{array}\right)\left(\begin{array}{cc}
1 & 0 \\
0 & -1
\end{array}\right)=\left(\begin{array}{cc}
-q_{2} & -p \\
* & q_{1}
\end{array}\right)
$$

from which one sees that $-L(p, q)=L(-p, q)$.

Lens spaces give examples of manifolds which are homotopy equivalent, but not homeomorphic [Bro60]. In fact, it is a highly non-trivial result that the only homeomorphisms between lens spaces are the "obvious" ones explained above.

Theorem 5.1 (Brody [Bro60]). Lens spaces $L\left(p_{1}, q_{1}\right)$ and $L\left(p_{2}, q_{2}\right)$ are homeomorphic if and only if $\left|p_{1}\right|=\left|p_{2}\right|$ and $q_{1} \equiv \pm q_{2}^{ \pm 1} \bmod p$.

Now consider a Seifert fiber space of the form $M\left(0 ;\left(\alpha_{1}, \beta_{1}\right),\left(\alpha_{2}, \beta_{2}\right)\right)$. This is constructed by gluing two standard fibered tori into a manifold with boundary $M_{0}=F_{0} \times S^{1}$ where $F_{0}$ is a sphere with two disks removed and so is an annulus. As $M_{0}$ is an $I$-bundle, this is effectively the same as gluing the boundaries of the two solid tori together by a linear map that respects the fiberings, and we may think of the Seifert fiber space as a fibered marked lens space.

Theorem 5.2. The Seifert invariant $\left(0 ;\left(\alpha_{1}, \beta_{1}\right),\left(\alpha_{2}, \beta_{2}\right)\right)$ yields a fibered marked lens space with gluing matrix

$$
\left(\begin{array}{cc}
-\alpha_{1} \beta_{2}^{\prime}-\alpha_{2}^{\prime} \beta_{1} & \alpha_{1} \beta_{2}+\alpha_{2} \beta_{1} \\
* & \alpha_{1}^{\prime} \beta_{2}+\alpha_{2} \beta_{1}^{\prime}
\end{array}\right) \quad \text { where }\left(\begin{array}{cc}
\alpha_{i} & \beta_{i} \\
\alpha_{i}^{\prime} & \beta_{i}^{\prime}
\end{array}\right) \in S L(2, \mathbb{Z})
$$

In particular, the marked lens space is $L(p, q)$ with

$$
p=\alpha_{1} \beta_{2}+\alpha_{2} \beta_{1} \text { and } q=\alpha_{1}^{\prime} \beta_{2}+\alpha_{2} \beta_{1}^{\prime} .
$$

Proof. Recall in the construction of a Seifert fiber space by gluing that

$$
\left(\begin{array}{c}
M_{i} \\
L_{i}
\end{array}\right)=\left(\begin{array}{cc}
\alpha_{i} & \beta_{i} \\
\alpha_{i}^{\prime} & \beta_{i}^{\prime}
\end{array}\right)\left(\begin{array}{c}
C_{i} \\
H_{i}
\end{array}\right)
$$

where $H_{i}$ is a vertical fiber, $C_{i}$ is a horizontal circle, and $\alpha_{i}^{\prime}$ and $\beta_{i}^{\prime}$ are chosen to yield so that the matrix lies in $S L(2, \mathbb{Z})$.

Note that we are not free to replace $L_{i}$ with $-L_{i}$ here without changing the gluing. Hence, the pair $\left(\alpha_{i}, \beta_{i}\right)$ in the Seifert invariant determines an orientation of the longitude $L_{i}$ and so also determines an orientation of the core of the solid torus. This means we are in fact constructing a marked lens space. 
As the two circles $C_{1}$ and $C_{2}$ are the boundaries of two disks punched out of a sphere, $C_{1} \sim-C_{2}$ in homology. As regular vertical fibers, $H_{1} \sim H_{2}$ in homology. Hence,

$$
\begin{aligned}
\left(\begin{array}{l}
C_{1} \\
H_{1}
\end{array}\right) & =\left(\begin{array}{cc}
-1 & 0 \\
0 & 1
\end{array}\right)\left(\begin{array}{l}
C_{2} \\
H_{2}
\end{array}\right) \Rightarrow \\
\left(\begin{array}{cc}
\alpha_{1} & \beta_{1} \\
\alpha_{1}^{\prime} & \beta_{1}^{\prime}
\end{array}\right)^{-1}\left(\begin{array}{c}
M_{1} \\
L_{1}
\end{array}\right) & =\left(\begin{array}{cc}
-1 & 0 \\
0 & 1
\end{array}\right)\left(\begin{array}{cc}
\alpha_{2} & \beta_{2} \\
\alpha_{2}^{\prime} & \beta_{2}^{\prime}
\end{array}\right)^{-1}\left(\begin{array}{c}
M_{2} \\
L_{2}
\end{array}\right) \Rightarrow \\
\left(\begin{array}{c}
M_{1} \\
L_{1}
\end{array}\right) & =\left(\begin{array}{cc}
\alpha_{1} & \beta_{1} \\
\alpha_{1}^{\prime} & \beta_{1}^{\prime}
\end{array}\right)\left(\begin{array}{cc}
-1 & 0 \\
0 & 1
\end{array}\right)\left(\begin{array}{ll}
-\beta_{2}^{\prime} & \beta_{2} \\
-\alpha_{2}^{\prime} & \alpha_{2}
\end{array}\right)\left(\begin{array}{c}
M_{2} \\
L_{2}
\end{array}\right) \Rightarrow \\
\left(\begin{array}{c}
M_{1} \\
L_{1}
\end{array}\right) & =\left(\begin{array}{cc}
-\alpha_{1} \beta_{2}^{\prime}-\alpha_{2}^{\prime} \beta_{1} & \alpha_{1} \beta_{2}+\alpha_{2} \beta_{1} \\
* & \alpha_{1}^{\prime} \beta_{2}+\alpha_{2} \beta_{1}^{\prime}
\end{array}\right)\left(\begin{array}{c}
M_{2} \\
L_{2}
\end{array}\right) .
\end{aligned}
$$

Using this, we may calculate which lens spaces arise as unit tangent bundles.

Corollary 5.3. If $\Sigma$ is a sphere with 0,1 , or 2 cone points added, then $U T \Sigma$ is a marked lens space of the form $L(p,-1)$ with $p \leq-2$.

Proof. By proposition 3.1 we may write the fibering as $M\left(0 ;\left(\alpha_{1},-1\right),\left(\alpha_{2},-1\right)\right)$ where $\alpha_{1}=\alpha_{2}=1$ if $\Sigma=S^{2}$ is the two sphere and $\alpha_{1}=1$ if $\Sigma$ has a single cone point. One can then apply theorem 5.2 with $\alpha_{i}^{\prime}=1$ and $\beta_{i}^{\prime}=0$ to get the result. Alternatively, it is fairly easy to calculate the gluing matrix directly. Adapting the proof of theorem 5.2, the matrix is given by

$$
\left(\begin{array}{cc}
\alpha_{2} & -1 \\
1 & 0
\end{array}\right)\left(\begin{array}{cc}
-1 & 0 \\
0 & 1
\end{array}\right)\left(\begin{array}{cc}
\alpha_{1} & -1 \\
1 & 0
\end{array}\right)^{-1}=\left(\begin{array}{cc}
1 & -\alpha_{1}-\alpha_{2} \\
* & -1
\end{array}\right)
$$

Since $\alpha_{1}, \alpha_{2} \geq 1$, it follows that $p=-\alpha_{1}-\alpha_{2} \leq-2$.

Proposition 5.4. Let $\hat{L}$ and $L$ be fibered marked lens spaces and let $\pi: \hat{L} \rightarrow L$ be a fiberwise covering of degree $d$. Then there are integers $p$ and $q$ such that $\hat{L}=$ $L(p, q)$ and $L=L(d p, q)$.

Proof. We use the same notation as in the proofs of propositions 3.3 and 3.5 . It follows from those proofs that $\pi\left(M_{i}\right)=m_{i}$ and $\pi\left(L_{i}+k_{i} M_{i}\right)=d \ell_{i}$ for some integer $k_{i}$. Then,

$$
\begin{aligned}
\pi\left(\begin{array}{c}
M_{1} \\
L_{1}
\end{array}\right) & =\left(\begin{array}{cc}
-q_{2} & p \\
* & q_{1}
\end{array}\right) \pi\left(\begin{array}{c}
M_{2} \\
L_{2}
\end{array}\right) \Rightarrow \\
\left(\begin{array}{cc}
1 & 0 \\
-k_{1} & d
\end{array}\right)\left(\begin{array}{l}
m_{1} \\
\ell_{1}
\end{array}\right) & =\left(\begin{array}{cc}
-q_{2} & p \\
* & q_{1}
\end{array}\right)\left(\begin{array}{cc}
1 & 0 \\
-k_{2} & d
\end{array}\right)\left(\begin{array}{c}
m_{2} \\
\ell_{2}
\end{array}\right) \Rightarrow \\
\left(\begin{array}{c}
m_{1} \\
\ell_{1}
\end{array}\right) & =\frac{1}{d}\left(\begin{array}{cc}
d & 0 \\
k_{1} & 1
\end{array}\right)\left(\begin{array}{cc}
-q_{2} & p \\
* & q_{1}
\end{array}\right)\left(\begin{array}{cc}
1 & 0 \\
-k_{2} & d
\end{array}\right)\left(\begin{array}{c}
m_{2} \\
\ell_{2}
\end{array}\right) \Rightarrow \\
\left(\begin{array}{c}
m_{1} \\
\ell_{1}
\end{array}\right) & =\left(\begin{array}{cc}
-q_{2}-k_{2} p & d p \\
* & q_{1}+k_{1} p
\end{array}\right)\left(\begin{array}{c}
m_{2} \\
\ell_{2}
\end{array}\right)
\end{aligned}
$$

Hence, the claim holds with $q=q_{1}+k_{1} p$. 
Note that $q \bmod d p$ uniquely determines $q \bmod p$, and so the covered lens space uniquely determines the covering lens space.

Proposition 5.5. A fibered marked lens space $L(p, q)$ has a horizontal vector field if and only if $p \neq 0$ and $q \equiv-1 \bmod p$.

Proof. First assume $L(p, q)$ has a horizontal vector field. Then by theorem 4.5 and corollary 5.3 it fiberwise covers $L(d p,-1)$ for some integer $d$ with the further property that $d p \leq-2$. This implies that $p$ must be non-zero. By proposition 5.4 $L(p, q)=L(p,-1)$ as a marked lens space and so $q \equiv-1 \bmod p$.

Conversely, assume $p \neq 0$ and $q \equiv-1 \bmod p$. As explained earlier in this section, we may make choices of longitudes, or equivalently choices of $\alpha_{i}^{\prime}$ and $\beta_{i}^{\prime}$, such that the gluing matrix is

$$
\left(\begin{array}{ll}
\alpha_{1} & \beta_{1} \\
\alpha_{1}^{\prime} & \beta_{1}^{\prime}
\end{array}\right)\left(\begin{array}{cc}
-1 & 0 \\
0 & 1
\end{array}\right)\left(\begin{array}{ll}
\alpha_{2} & \beta_{2} \\
\alpha_{2}^{\prime} & \beta_{2}^{\prime}
\end{array}\right)^{-1}=\left(\begin{array}{cc}
1 & p \\
* & -1
\end{array}\right) .
$$

Then $\alpha_{1} \beta_{1}^{\prime}-\alpha_{1}^{\prime} \beta_{1}=1$ as it is the determinant of a matrix in $S L(2, \mathbb{R})$. Theorem 5.2 implies that $\alpha_{1}^{\prime} \beta_{2}+\alpha_{2} \beta_{1}^{\prime}=-1$. These equations may be rewritten as

$$
\alpha_{1}^{\prime}\left(\frac{-\beta_{1}}{\alpha_{1}}\right)=\frac{1}{\alpha_{1}}-\beta_{1}^{\prime} \quad \text { and } \quad \alpha_{1}^{\prime}\left(\frac{-\beta_{2}}{\alpha_{2}}\right)=\frac{1}{\alpha_{2}}+\beta_{1}^{\prime} .
$$

One may then show that proposition 3.6 holds with $d=\alpha_{1}^{\prime}$. Indeed, $d\left(-\beta_{i} / \alpha_{i}\right) \equiv$ $1 / \alpha_{i} \bmod \mathbb{Z}$ since $\beta_{1}^{\prime}$ is an integer, and summing the above equations gives

$$
d \cdot e(M \rightarrow \Sigma)=d\left(-\frac{\beta_{1}}{\alpha_{1}}-\frac{\beta_{2}}{\alpha_{2}}\right)=\frac{1}{\alpha_{1}}+\frac{1}{\alpha_{2}}=\chi(\Sigma) .
$$

Before proceeding, we attempt here to give a more intuitive and topological explanation of why $q \equiv-1 \bmod p$ is a necessary and sufficient condition for a horizontal vector field and why $d=\alpha_{1}^{\prime}$ in the proof above. Since the results have already been rigorously proved above, we do not give complete details in the following explanation.

First, suppose that we have Seifert fibering on a marked lens space such that the gluing torus is a union of fibers and that there is a horizontal vector field. For any closed curve on the gluing torus, we can measure how many turns the horizontal vector field makes as we travel around this curve. This may be done, say, by counting how many times the vector field crosses the gluing torus and changes from pointing into one solid torus to pointing into the other. This counting of turns induces a group homomorphism $\phi: H_{1}(T) \rightarrow \mathbb{Z}$ where $H_{1}(T)$ is the first homology group of the gluing torus. Since $M_{i}$ bounds a meridional disk and the horizontal vector field projects to a non-zero vector field on the disk, the Poincaré-Hopf theorem implies that the vector field must make exactly one clockwise turn when going around $M_{i}$. Hence $\phi\left(M_{i}\right)=-1$. In fact, this is only restriction on $\phi$. One can check that for any horizontal vector field defined on the boundary of a standard fibered torus, that so long as $\phi\left(M_{i}\right)=-1$ it is possible to extend the horizontal vector field to all of the solid torus. 
The relation

$$
\left(\begin{array}{c}
M_{1} \\
L_{1}
\end{array}\right)=\left(\begin{array}{cc}
-q_{2} & p \\
* & q_{1}
\end{array}\right)\left(\begin{array}{c}
M_{2} \\
L_{2}
\end{array}\right)
$$

implies that $M_{1} \sim-q_{2} M_{2}+p L_{1}$ and so

$$
-1=\phi\left(M_{1}\right)=-q_{2} \phi\left(M_{2}\right)+p \phi\left(L_{1}\right)=q_{2}+p \phi\left(L_{1}\right) .
$$

That is, $q_{2} \equiv-1 \bmod p$ and the marked lens space is $L(p,-1)$.

Conversely, suppose we have a Seifert fibering on $L(p,-1)$ and we wish to construct a horizontal vector field. It is enough to find a homomorphism $\phi$ : $H_{1}(T) \rightarrow \mathbb{Z}$ such that $\phi\left(M_{1}\right)=\phi\left(M_{2}\right)=-1$. Up to choosing different longitudes on the solid tori, we may assume that the gluing matrix is of the form

$$
\left(\begin{array}{c}
M_{1} \\
L_{1}
\end{array}\right)=\left(\begin{array}{cc}
1 & p \\
0 & -1
\end{array}\right)\left(\begin{array}{c}
M_{2} \\
L_{2}
\end{array}\right) \text {. }
$$

Under such a choice, we may then define $\phi$ by $\phi\left(M_{i}\right)=-1$ and $\phi\left(L_{i}\right)=0$ for $i=1,2$ and see that this agrees with the gluing. In the construction of a Seifert fibering by gluing, the cycles in homology satisfy

$$
\left(\begin{array}{c}
C_{i} \\
H_{i}
\end{array}\right)=\left(\begin{array}{ll}
\alpha_{i} & \beta_{i} \\
\alpha_{i}^{\prime} & \beta_{i}^{\prime}
\end{array}\right)^{-1}\left(\begin{array}{c}
M_{i} \\
L_{i}
\end{array}\right)=\left(\begin{array}{cc}
\beta_{i}^{\prime} & -\beta_{i} \\
-\alpha_{i}^{\prime} & \alpha_{i}
\end{array}\right)\left(\begin{array}{c}
M_{i} \\
L_{i}
\end{array}\right)
$$

and so $\phi\left(H_{i}\right)=-\alpha_{i}^{\prime} \phi\left(M_{i}\right)+\alpha_{i} \phi\left(L_{i}\right)=\alpha_{i}^{\prime}$. Since $H_{i}$ is a regular fiber, $\phi\left(H_{i}\right)$ measures the number of twists the vector field makes around the regular fiber and so equals the degree $d$ of the covering $M \rightarrow U T \Sigma$. This shows that $d=\alpha_{1}^{\prime}=\alpha_{2}^{\prime}$. This ends our discussion of the proof of proposition 5.5 .

We now prove a version of theorem 1.1 for fibered marked lens spaces.

Proposition 5.6. Suppose $M$ is diffeomorphic to the lens space $L(p, q)$ with $p \geq 0$ and consider only the Seifert fiberings on $M$ which have an invariant of the form $\left(0 ;\left(\alpha_{1}, \beta_{1}\right),\left(\alpha_{2}, \beta_{2}\right)\right)$.

(1) If $p=1$ or $p=2$, then every such fibering on $M$ has a horizontal vector field.

(2) If $p \geq 3$ and $q \equiv \pm 1 \bmod p$, then $M$ has infinitely many fiberings of this form which support horizontal vector fields and infinitely many which do not.

(3) For all other cases of $p$ and $q$, no fibering of this form on $M$ has a horizontal vector field.

Proof. We prove this using theorem 5.1 and proposition 5.5, If $p=0$, then the only marked lens space diffeomorphic to $M$ is $L(0,1)=S^{2} \times S^{1}$ and proposition 5.5implies that no fibering has a horizontal vector field.

If $p=1$ or $p=2$, the fact that $q$ is coprime to $p$ implies that $q \equiv-1 \bmod p$. That is, the only marked lens space diffeomorphic to $M$ is $L(p,-1)$ and so every fibering has a horizontal vector field. 
If $M$ is diffeomorphic to $L(p, q)$ with $|p|>3$ and $q \equiv-1 \bmod p$, then $M$ is diffeomorphic to both $L(p,-1)$ and $L(p,+1)$. The former has infinitely many fiberings, all of which have a horizontal vector field; the latter has infinitely many fiberings, none of which has a horizontal vector field.

Finally, if $q \not \equiv \pm 1 \bmod p$, then theorem 5 .1 shows that $M$ is not diffeomorphic to $L(p,-1)$ and so no fibering of the form $\left(0 ;\left(\alpha_{1}, \beta_{1}\right),\left(\alpha_{2}, \beta_{2}\right)\right)$ will have a horizontal vector field.

5.2. Other fiberings of lens spaces. To complete the proof of theorem 1.1 we must consider all possible Seifert fiberings of lens spaces. To do this, we first list out all closed 3-manifolds which have multiple possible Seifert fiberings.

Proposition 5.7. A Seifert fibering of an oriented 3-manifold (without boundary) is unique up to isomorphism with the following exceptions:

(1) Every lens space has infinitely many different Seifert fiberings, and any Seifert fibering of a lens space is either of the form

$$
\left(0 ;\left(\alpha_{1}, \beta_{1}\right),\left(\alpha_{2}, \beta_{2}\right)\right) \quad \text { or }(-1 ;(\alpha, \pm 1)) .
$$

(2) For a non-zero rational number p/q written in lowest terms, write $\alpha_{1}=$ $|p|, \alpha_{3}=|q|$, and define $\beta_{1}$ and $\beta_{3}$ such that $\beta_{3} / \alpha_{3}=\alpha_{1} / \beta_{1}=p / q$, then the invariants

$$
\left(0 ;(2,1),(2,-1),\left(\alpha_{3}, \beta_{3}\right)\right) \text { and }\left(-1 ;\left(\alpha_{1}, \beta_{1}\right)\right)
$$

give fiberings of the same manifold. This manifold is a lens space if and only if $\alpha_{3}=1$.

(3) The unit tangent bundles of the 2222 orbifold and the Klein bottle are diffeomorphic as manifolds.

Proof. This is a re-statement of a known result on the uniqueness of Seifert fiberings. As a reference, we give the lecture notes of Hatcher [Hat07, Theorem 2.3]. The theorem as stated there has five cases which we quote here using the notation conventions from those notes:

(a) $M(0,1 ; \alpha / \beta)$, the various model Seifert fiberings of $S^{1} \times D^{2}$.

(b) $M(0,1 ; 1 / 2,1 / 2)=M(-1,1 ;)$, two fiberings of $S^{1} \widetilde{\times} S^{1} \widetilde{\times} I$.

(c) $M\left(0,0 ; \alpha_{1} / \beta_{1}, \alpha_{2} / \beta_{2}\right)$, various fiberings of $S^{3}, S^{1} \times S^{2}$ and lens spaces.

(d) $M(0,0 ; 1 / 2,-1 / 2, \alpha / \beta)=M(-1,0 ; \beta / \alpha)$ for $\alpha, \beta \neq 0$.

(e) $M(0,0 ; 1 / 2,1 / 2,-1 / 2,-1 / 2)=M(-2,0 ;)$, two fiberings of $S^{1} \widetilde{\times} S^{1} \widetilde{\times} S^{1}$.

Cases (a) and (b) are for manifolds with boundary and can be ignored here. We have used proposition 3.1 above to rephrase case (e) in terms of unit tangent bundles. Since the manifold in case (e) is finitely covered by the 3-torus, one can show that it does not overlap with cases (c) or (d). Assuming for simplicity that $\alpha$ and $\beta$ are positive, the invariants in case (d) may be written in our notation as

$$
\left(0 ;(2,1),(2,-1),\left(\alpha_{3}, \beta_{3}\right)\right) \text { and }\left(-1 ;\left(\alpha_{1}, \beta_{1}\right)\right)
$$


where $\alpha / \beta=\beta_{1} / \alpha_{1}=\alpha_{3} / \beta_{3}$ and $\alpha_{i}>0$. Note that if $\alpha_{3}=1$, then

$$
M\left(0 ;(2,1),(2,-1),\left(1, \beta_{3}\right)\right)=M\left(0 ;(2,-1),\left(2,2 \beta_{3}-1\right)\right)
$$

is a fibered marked lens space, and so there is actually some overlap between cases (c) and (d). Using the formula in [JN83, \$6], the fundamental group of the manifold $M\left(-1 ;\left(\alpha_{1}, \beta_{1}\right)\right)$ is given by

$$
\left\langle a, q, h: a^{-1} h a=h^{-1},[h, q]=1, q^{\alpha_{1}} h^{\beta_{1}}=1, q a^{2}=1\right\rangle
$$

or equivalently

$$
\left\langle a, h: a^{-1} h a=h^{-1}, a^{2 \alpha_{1}}=h^{\beta_{1}}\right\rangle .
$$

If $\left|\beta_{1}\right|>1$, this group is non-cyclic. and the manifold is not a lens space. (In fact, the manifold is a prism manifold.)

Hence, the only fiberings on lens spaces left to study are those of the form $(-1 ;(\alpha, \pm 1))$. Up to a choice of orientation, these are all unit tangent bundles.

Proposition 5.8. Let $M$ be diffeomorphic to a lens space and suppose $M$ is Seifert fibered, but is not a fibered marked lens space. Then (up to a change of orientation) there is $\alpha \geq 1$ such that $M$ is unit tangent bundle of the $\alpha \times$ orbifold, $M$ is diffeomorphic to $L(4 \alpha, 2 \alpha \pm 1)$, and the fibering has a horizontal vector field.

Remark. Recall from the orbifold notation introduced in section 2 that the $\alpha \times$ orbifold is $\mathbb{R} P^{2}$ with a cone point of order $\alpha$ added. If $\alpha=1$, this orbifold is $\mathbb{R} P^{2}$ itself.

Proof. By proposition 5.7, the fibering must have Seifert invariant $(-1 ;(\alpha, \pm 1))$. By corollary 3.2. up to a possible change of orientation we may assume that the invariant is $(-1 ;(\alpha,-1))$. Proposition 3.1 then implies that $M$ is the unit tangent bundle of the $\alpha \times$ orbifold and theorem 4.5 shows that it has a horizontal vector field. By proposition 5.7, this manifold is diffeomorphic to

$$
M(0 ;(2,1),(2,-1),(1, \alpha))=M(0 ;(2,1),(2,2 \alpha-1)) .
$$

Using theorem 5.2 or directly from the computation

$$
\left(\begin{array}{cc}
2 & 2 \alpha-1 \\
1 & \alpha
\end{array}\right)\left(\begin{array}{cc}
-1 & 0 \\
0 & 1
\end{array}\right)\left(\begin{array}{ll}
2 & 1 \\
1 & 1
\end{array}\right)^{-1}=\left(\begin{array}{cc}
-(2 \alpha+1) & 4 \alpha \\
* & 2 \alpha+1
\end{array}\right)
$$

one may show that this lens space is $L(4 \alpha, 2 \alpha+1)$. By theorem $5.1, L(p, q)$ is diffeomorphic to $M$ if and only if $|p|=4 \alpha$ and $q \equiv 2 \alpha \pm 1 \bmod p$.

This shows that beyond the fibered marked lens spaces, there is exactly one additional Seifert fibering on $L(4 \alpha, 2 \alpha \pm 1)$ for each $\alpha \geq 1$. Theorem 1.1 now follows as a combination of propositions 5.6 and 5.8 .

\section{ELLIPTIC ORBIFOLDS}

We now consider Seifert fiberings over elliptic orbifolds. We start by listing out all of the possible orbifolds. 
Proposition 6.1. An elliptic orbifold is of one of the following forms:
(1) the $p p$ orbifold with $p \geq 1$;
(3) the $23 q$ orbifold with $3 \leq q \leq 5$;

(2) the $22 p$ orbifold with $p \geq 2$;

(4) the $p \times$ orbifold with $p \geq 1$.

Note that the 2-sphere and real projective plane occur as special cases with $p=1$. For further details and a proof of this result, see the section of [Sco83] entitled "The geometry of $S^{2} \times \mathbb{R}$ ” and the references therein.

Proposition 6.2. Let $M \rightarrow \Sigma$ be a Seifert fibering over an elliptic base orbifold. Then $M$ is diffeomorphic to a lens space if and only if either

(1) $\Sigma$ is the $p p$ orbifold with $p \geq 1$; or

(2) $M= \pm U T \Sigma$ where $\Sigma$ is the $p \times$ orbifold with $p \geq 1$.

Proof. If $\Sigma$ is the $p p$ orbifold, the Seifert invariant may be written as

$$
\left(g ;\left(\alpha_{1}, \beta_{1}\right),\left(\alpha_{1}, \beta_{2}\right)\right)
$$

with $\alpha_{1}=\alpha_{2}=p$ and so $M$ is a fibered marked lens space. If $\Sigma$ is the $p \times$ orbifold, then propositions 5.7 and 5.8 imply that $M$ is diffeomorphic to a lens space if and only if $M= \pm U T \Sigma$. The other elliptic orbifolds listed in proposition 6.1 may be ruled out by proposition 5.7

Now, consider Seifert fiber spaces $M_{1}$ and $M_{2}$ over an orbifold $\Sigma$ and suppose there is a fiberwise covering $M_{1} \rightarrow M_{2}$ of degree $d>1$. Corollary 3.7 states that $d \cdot e\left(M_{1} \rightarrow \Sigma\right)=e\left(M_{2} \rightarrow \Sigma\right)$. Roughly speaking, the Euler number measures the amount of "twisting" of the fibering, and the effect of quotienting $M_{1}$ down to $M_{2}$ is to increase this twisting by a factor of $d$. Conversely, if we consider $M_{2}$ first, finding a fiberwise covering map $M_{1} \rightarrow M_{2}$ is in some sense equivalent to finding a way to reduce the twisting of the fibering by a factor of $d$. If we know, however, that the fibering $M_{2} \rightarrow \Sigma$ already has the minimum possible amount of (non-zero) twisting for a fibering over $\Sigma$, then it cannot be fiberwise covered by another fibering.

This is the technique we will use to prove theorem 1.2. We will show for all but one of the cases that $e(U T \Sigma \rightarrow \Sigma)$ is the smallest possible positive Euler number for any Seifert fibering over $\Sigma$. In the exceptional case, a degree two covering is possible, but $M$ must be a lens space.

Proposition 6.3. Suppose $\Sigma$ is an elliptic orbifold and $M \rightarrow U T \Sigma$ is a fiberwise covering of positive degree $d$. Then the degreed is at most two. Moreover, the $d=2$ case occurs exactly when $M$ is of the form $M\left(0 ;\left(\alpha, \beta_{1}\right),\left(\alpha, \beta_{2}\right)\right)$ where $\alpha \geq 1$ is odd,

$$
\beta_{1}=\frac{\alpha}{2}-\frac{1}{2} \text { and } \beta_{2}=-\frac{\alpha}{2}-\frac{1}{2} \text {. }
$$

The two-fold covering above with $\alpha=1$ corresponds to the so-called "belt trick" or "plate trick" where $S^{3}$ double-covers $U T S^{2}$.

Proof. We proceed through the cases in proposition 6.1 in an order which makes the overall proof easiest to follow. 
Case $1 . \Sigma$ is the $23 q$ orbifold for $3 \leq q \leq 5$.

We give a full proof for the 235 orbifold. Here $e(U T \Sigma \rightarrow \Sigma)=\chi(\Sigma)=1 / 30$ and $M$ has a Seifert invariant which may be written as $\left(0 ;\left(2, \beta_{1}\right),\left(3, \beta_{2}\right),\left(5, \beta_{3}\right)\right)$ for some values of $\beta_{i}$. The Euler number of $M \rightarrow \Sigma$ is a linear combination of $1 / 2$, $1 / 3$ and $1 / 5$ with integer coefficients and hence must be an integer multiple of $1 / 30$. Say $k$ is such that $e(M \rightarrow \Sigma)=k / 30$. Then $d \cdot e(M \rightarrow \Sigma)=e(U T \Sigma \rightarrow \Sigma)$ implies that $d k=1$. As $d$ and $k$ are integers and $d$ is positive, the only possibility is that $d=k=1$. The 233 and 234 orbifolds have similar proofs with $1 / 6$ and $1 / 12$ replacing $1 / 30$ above.

Case $2 . \Sigma$ is the $22 p$ orbifold where $p$ is even.

Here $e(U T \Sigma \rightarrow \Sigma)=\chi(\Sigma)=1 / p$ and the Euler number of $M \rightarrow \Sigma$ is a linear combination of $1 / 2$ and $1 / p$ with integer coefficients. Hence, $e(M \rightarrow \Sigma)=k / p$ for some integer $k$ and $d k=1$ implies that $d=k=1$.

Case $3 . \Sigma$ is the $22 p$ orbifold where $p$ is odd.

As in the previous case, $e(U T \Sigma \rightarrow \Sigma)=\chi(\Sigma)=1 / p$ and the Euler number of $M \rightarrow \Sigma$ is a linear combination of $1 / 2$ and $1 / p$ with integer coefficients. However, as $p$ is odd, we may only conclude that $e(M \rightarrow \Sigma)=k / 2 p$ for some integer $k$ and so $d k=2$. As shown by proposition 3.3 , when quotienting along fibers to produce a $d$-fold cover, the integer $d$ must be coprime to the orders of all of the cone points. As $\Sigma$ has cone points of order $2, d$ cannot be even and so $d=1$ is the only possibility.

Case $4 . \Sigma$ is the $p \times$ orbifold.

Here $e(U T \Sigma \rightarrow \Sigma)=\chi(\Sigma)=1 / p$ and $M$ has a Seifert invariant which may be written as $\left(-1 ;\left(p, \beta_{1}\right)\right)$ for some value of $\beta_{1}$. Hence, $e(M \rightarrow \Sigma)=k / p$ for some integer $k$ and $d k=1$ implies that $d=k=1$.

Case 5. $\Sigma$ is the $p p$ orbifold where $p$ is even.

Here $e(U T \Sigma \rightarrow \Sigma)=\chi(\Sigma)=2 / p$ and $e(M \rightarrow \Sigma)=k / p$ for some integer $k$. Hence, $d k=2$. By proposition 3.3, $d$ is coprime to $p$ and so must be odd. This is only possible if $d=1$.

Case 6. $\Sigma$ is the $p p$ orbifold where $p$ is odd.

As in the previous case, $d k=2$ where $d$ is coprime to $p$. As $p$ is odd, both $d=1$ and $d=2$ are possible. Assume $d=2$ for the rest of the proof. Case (4) of theorem 4.5 holds with $d=2$ and $\alpha=\alpha_{1}=\alpha_{2}=p$, showing that

$$
d \cdot e(M \rightarrow \Sigma)=-\frac{2}{\alpha}\left(\beta_{1}+\beta_{2}\right)=\frac{2}{\alpha}=\chi(\Sigma) \quad \text { and } \quad 2 \beta_{i} / \alpha \equiv-1 / \alpha \bmod \mathbb{Z} .
$$

As $\alpha$ is odd, it follows that $\beta_{i} \equiv \frac{1}{2}(\alpha-1) \bmod \alpha$. Using the allowed manipulations on Seifert invariants, we may adjust the ratios $\beta_{i} / \alpha_{i}$ by integer amounts and assume with loss of generality that $\beta_{1}=\frac{\alpha}{2}-\frac{1}{2}$. Then $\beta_{1}+\beta_{2}=-1$ implies that $\beta_{2}=-\frac{\alpha}{2}-\frac{1}{2}$.

Proving theorem 1.2 is now a matter of combining the above results. 
Proof of theorem 1.2. By theorem 4.5, having a horizontal vector field is equivalent to having a fiberwise covering $M \rightarrow U T \Sigma$ of some degree $d$. Up to changing the orientation of $M$, we may assume $d \geq 1$. Since we are assuming $M$ is not diffeomorphic to a lens space, propositions 6.2 and 6.3 imply that $d=1$ and so $M=U T \Sigma$.

As a final observation, note that for each $p>1$, the unit tangent bundle of the $22 p$ orbifold has two fiberings

$$
M(0 ;(2,1),(2,-1),(p,-1) \text { and } M(-1 ;(1,-p))
$$

given by item (2) of proposition 5.7. The first of these fibering is the standard fibering of the unit tangent bundle, and so has a horizontal vector field. The second is a fibering over the real projective plane and since $p>\chi\left(\mathbb{R} P^{2}\right)=1$, this fibering does not have a horizontal vector field.

\section{PARABOLIC ORBIFOLDS}

We now consider Seifert fiberings over elliptic orbifolds. There are exactly seven orbifolds of this type.

Proposition 7.1. The parabolic orbifolds are as follows:

(1) the 2-torus $\mathbb{T}^{2}$;

(2) the Klein bottle K;

(3) the 236 orbifold;

(4) the 244 orbifold;
(5) the 333 orbifold;

(6) the 2222 orbifold;

(7) the $22 \times$ orbifold.

For further details and a proof of this result, see the section of [Sco83] entitled “The geometry of $E^{3}$ ” and the references therein. See also $\$ 2.1$ of [Hat07|. It is actually a somewhat enjoyable exercise to prove the result directly by starting with a surface $\Sigma_{0}$ with $\chi\left(\Sigma_{0}\right) \geq 0$ and seeing which combinations of cone points may be added to get $\chi(\Sigma)$ down to exactly zero.

7.1. Self-coverings. For each of the parabolic orbifolds, $U T \Sigma$ is an oriented Seifert fiber space and corollary 3.2 implies that $e(U T \Sigma \rightarrow \Sigma)=\chi(\Sigma)=0$. It turns out that, up to orientation, these unit tangent bundles are the only Seifert fiber spaces of this form.

Proposition 7.2. There are exactly ten distinct Seifert fiberings $M \rightarrow \Sigma$ for which $M$ is oriented and $e(M \rightarrow \Sigma)=\chi(\Sigma)=0$. These are all of the form $M= \pm U T \Sigma$. Moreover, if $\Sigma$ is the 236, 244, or 333 orbifold, then $U T \Sigma \neq-U T \Sigma$. For the other parabolic orbifolds, $U T \Sigma=-U T \Sigma$.

Proof. A list of these Seifert invariants appears in a number of places. For instance, $\$ 8.2$ of [Orl72] and the sections of [Sc083] and [Hat07] mentioned above. As the result is not difficult, we outline the proofs for two of the cases here and leave the others, which are similar, to the reader. 
The 236 orbifold. Consider a Seifert fibering $M \rightarrow \Sigma$ having invariant

$$
\left(0 ;\left(2, \beta_{1}\right),\left(3, \beta_{2}\right),\left(6, \beta_{3}\right)\right)
$$

and $e(M \rightarrow \Sigma)=0$. As $\beta_{2}$ is coprime to 3 , we have either $\beta_{2} \equiv 1$ or $\beta_{2} \equiv-1 \bmod 3$. By reversing the orientation of the manifold if necessary and using proposition 3.5, we may assume the latter holds. By doing valid operations on the Seifert invariant, adding an integer to one ratio $\beta_{i} / \alpha_{i}$ and subtracting it to another we may reduce to the case where the invariant is $\left(0 ;(2,-1),(3,-1),\left(6, \beta_{3}\right)\right.$. Then $e(M \rightarrow \Sigma)=0$ implies that $\beta_{3}=5$ and one may verify $M=U T \Sigma$.

The 333 orbifold. Consider a Seifert fibering $M \rightarrow \Sigma$ having invariant

$$
\left(0 ;\left(3, \beta_{1}\right),\left(3, \beta_{2}\right),\left(3, \beta_{3}\right)\right)
$$

and $e(M \rightarrow \Sigma)=0$. Each $\beta_{i}$ is coprime to 3 . One can show that $\beta_{1} \equiv \beta_{2} \equiv \beta_{3} \bmod$ 3 as otherwise $e(M \rightarrow \Sigma)$ would not be an integer. Applying proposition 3.5 if necessary, we may assume $\beta_{1} \equiv \beta_{2} \equiv \beta_{3} \equiv-1$. Similar to above, we may reduce to the case where the invariant is $\left(0 ;(3,-1),(3,-1),\left(3, \beta_{3}\right)\right.$. Then $e(M \rightarrow \Sigma)=0$ implies that $\beta_{3}=2$ and $M=U T \Sigma$.

Using proposition 3.5 , one can determine whether or not $U T \Sigma$ and $-U T \Sigma$ have the same Seifert invariants.

Proposition 7.3. Suppose $\Sigma$ is an elliptic orbifold with a (possibly empty) set of cone points of orders $\alpha_{1}, \ldots, \alpha_{n}$. If $M \rightarrow U T \Sigma$ is a fiberwise cover, then $M= \pm U T \Sigma$. Moreover, such a fiberwise cover of degree d exists if and only ifd is coprime to all of the $\alpha_{i}$.

Proof. First, suppose $M_{1} \rightarrow U T \Sigma$ is a fiberwise covering. Then, corollary $3.7 \mathrm{im}$ plies that $d \cdot e\left(M_{1} \rightarrow \Sigma\right)=\chi(\Sigma)=0$ and so $e\left(M_{1} \rightarrow \Sigma\right)=0$. As $M_{1}$ has the same base orbifold as $U T \Sigma$, proposition 7.2 applies and $M_{1}= \pm U T \Sigma$.

Conversely, starting from an integer $d$ coprime to all $\alpha_{i}$, proposition 3.6 gives a fiberwise covering $U T \Sigma \rightarrow M_{2}$. Corollary 3.7 implies that $e\left(M_{2} \rightarrow \Sigma\right)=0$ and then proposition 7.2 implies that $M_{2}= \pm U T \Sigma$. Hence, we have constructed a covering $U T \Sigma \rightarrow \pm U T \Sigma$. Up to a possible change of orientation, this gives a covering $\pm U T \Sigma \rightarrow U T \Sigma$ as desired.

Using the above propositions, it is a straightforward task to list out all of the possible coverings:

(1) If $\Sigma=\mathbb{T}^{2}$, then $U T \Sigma \cong-U T \Sigma$ and has a $d$-fold self-covering for all $d \neq 0$.

(2) If $\Sigma=K$ is the Klein bottle, then $U T \Sigma \cong-U T \Sigma$ and has a $d$-fold self-covering for all $d \neq 0$.

(3) If $\Sigma$ is the 236 orbifold, then $U T \Sigma d$-fold covers $U T \Sigma$ if $d \equiv+1 \bmod 6$, and $U T \Sigma d$-fold covers $-U T \Sigma$ if $d \equiv-1 \bmod 6$. 
(4) If $\Sigma$ is the 244 orbifold, then

$U T \Sigma d$-fold covers $U T \Sigma$ if $d \equiv+1 \bmod 4$, and

$U T \Sigma d$-fold covers $-U T \Sigma$ if $d \equiv-1 \bmod 4$.

(5) If $\Sigma$ is the 333 orbifold, then

$U T \Sigma d$-fold covers $U T \Sigma$ if $d \equiv+1 \bmod 3$, and

$U T \Sigma d$-fold covers $-U T \Sigma$ if $d \equiv-1 \bmod 3$.

(6) If $\Sigma$ is the 2222 orbifold, then

$U T \Sigma \cong-U T \Sigma$ and $d$-fold covers itself if $d \equiv 1 \bmod 2$.

(7) If $\Sigma$ is the $22 \times$ orbifold, then

$U T \Sigma \cong-U T \Sigma$ and $d$-fold covers itself if $d \equiv 1 \bmod 2$.

We now attempt to impart some intuition about the structure of these selfcoverings and their associated horizontal vector fields. Every parabolic orbifold is the quotient of $\mathbb{R}^{2}$ by a wallpaper group consisting of affine isometries. Consider the unit tangent bundle $U T \mathbb{R}^{2}$ of the Euclidean plane. An element of $U T \mathbb{R}^{2} \cong \mathbb{R}^{2} \times \mathbb{S}^{1}$ may be represented by a pair $(x, \theta)$ where $x \in \mathbb{R}^{2}$ and $\theta$ is an angle. Then $U T \mathbb{R}^{2}$ has self-covering maps of the form $(x, \theta) \mapsto(x, d \theta)$ for $d \geq 1$.

If $A: \mathbb{R}^{2} \rightarrow \mathbb{R}^{2}$ is an orientation-preserving isometry, then its derivative is of the form $(x, \theta) \mapsto\left(A(x), \theta+\theta_{0}\right)$ for some constant $\theta_{0}$. In some cases, this will commute with the $d$-fold self-covering; for instance, when $A$ is a rotation by an angle $\theta_{0}=\frac{2 \pi}{k}$ and $d \equiv 1 \bmod k$. If the self-covering commutes with every element of the wallpaper group, then this defines a self-covering map on unit tangent bundle of the orbifold.

As a concrete example, consider the wallpaper group associated to the 236 orbifold. This group is generated by three rotations of orders 2,3 , and 6 as depicted in fig. 1. If $d \equiv 1 \bmod 6$, the self-covering on $U T \mathbb{R}^{2}$ will quotient to the unit tangent bundle $U T \Sigma$.

7.2. Vector fields on surfaces. To completely handle Seifert fiberings over parabolic orbifolds, we must also consider case (1) of proposition 4.4 ,

Proposition 7.4. Let $\Sigma$ be the 2-torus or Klein bottle. Then any Seifert fibering $M \rightarrow \Sigma$ has a horizontal vector field (whether or not $M$ is orientable).

Proof. On such a surface, one can construct a unit vector field $v: \Sigma \rightarrow U T \Sigma$. Define $u: \Sigma \rightarrow U T \Sigma$ as the composition of $v$ with the projection $\pi: M \rightarrow \Sigma$ defining the Seifert fibering. Then, a horizontal vector field exists by proposition 4.1

For each of $\mathbb{T}^{2}$ and $K$, there are infinitely many oriented Seifert fiberings, one for each integer $e(M \rightarrow \Sigma)$. These manifolds have Euclidean geometry if $e(M \rightarrow \Sigma)=0$ and Nil geometry otherwise [Sc083]. There are exactly four nonorientable 3-manifolds with Euclidean geometry. (See \$8.2 of [Orl72].) Each of these has a Seifert fibering over $K$ and therefore has a horizontal vector field. Two of these manifolds also have Seifert fiberings over $\mathbb{T}^{2}$ which therefore also have horizontal vector fields. 


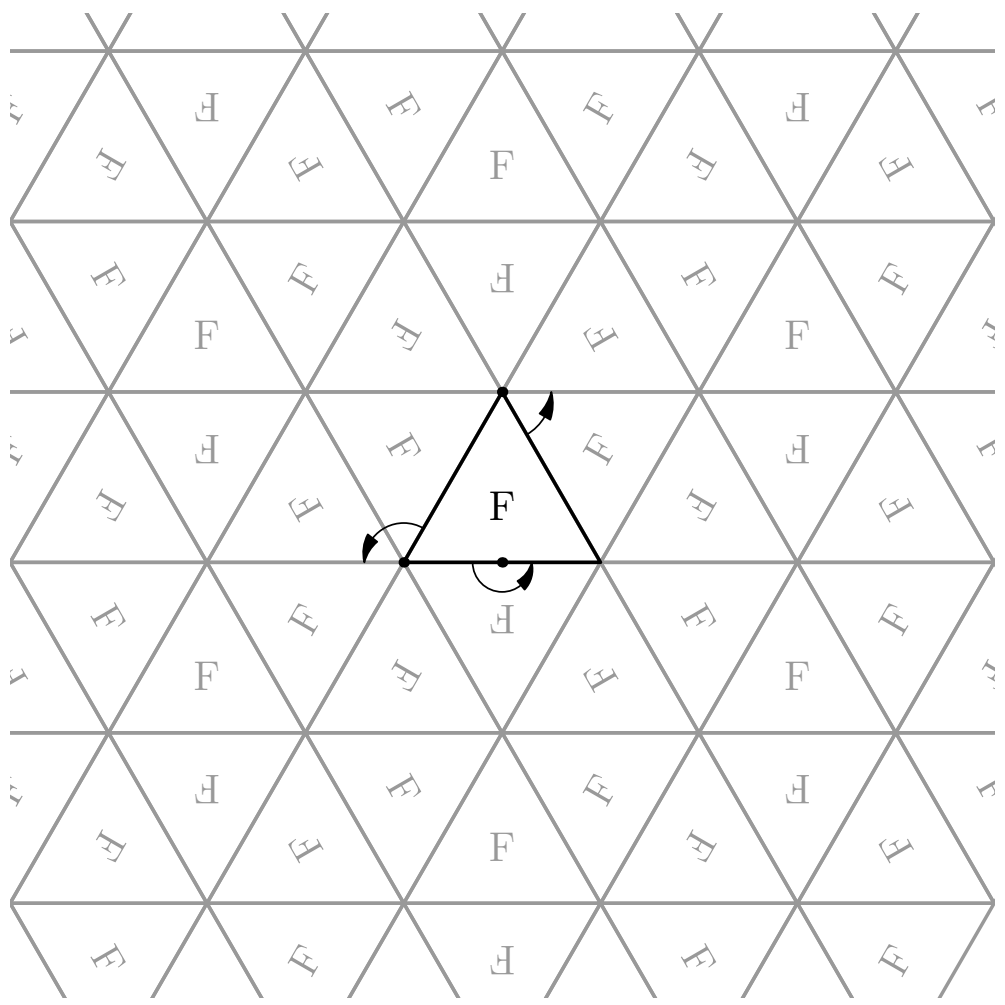

FIGURE 1. Generators of the wallpaper group associated to the 236 orbifold.

Proof of theorem 1.3, If $\Sigma$ is a surface with $\chi(\Sigma)=0$, then proposition 7.4 establishes the theorem, so suppose $\Sigma$ is not a surface. Theorem 4.5 and proposition 7.3 then imply that

$M$ has a horizontal vector field $\Leftrightarrow M$ fiberwise covers $U T \Sigma \Leftrightarrow M= \pm U T \Sigma$.

\section{HyPERBOLIC ORBIFOLDS}

The final class of orbifolds to consider are those with hyperbolic geometry. Even though this is the most general case, there is the least to say here. In this setting, it is relatively easy to find fiberwise coverings $M \rightarrow U T \Sigma$ of degree $d>1$. For circle bundles, if $\Sigma$ is a surface of genus $g \geq 2$, then there is a cover of degree $d$ for every factor $d$ of $\chi(g)=2-2 g$. Even for orbifolds without handles, non-trivial covers are possible. For instance, using theorem 4.5, one may show that

$$
M(0 ;(1,-1),(5,2),(5,2),(5,2))
$$

double covers the unit tangent bundle of the 555 orbifold.

For certain choices of cone points, there may be no non-trivial covers. For instance, if $\Sigma$ is the 237 orbifold, one may show that only $d= \pm 1$ is possible. The proof is of the same form as Case 1 of the proof of proposition 6.3 . 
If $\Sigma$ is a hyperbolic orbifold, the geodesic flow on $U T \Sigma$ is an Anosov flow and the flow is generated by a vector field which is horizontal. This flow lifts to any finite cover and is still Anosov on the cover. Ghys and Barbot showed that (up to orbit equivalency) every Anosov flow on a Seifert fiber space is of this form Ghy84, Bar96]. This establishes the equivalence (2) $\Leftrightarrow$ (3) in theorem 1.4. The equivalence $(1) \Leftrightarrow(2)$ is a re-statement of the results in section 4 .

\section{HOMOTOPIES OF HORIZONTAL VECTOR FIELDS}

For a given Seifert fibering $M \rightarrow \Sigma$, consider the space of all horizontal vector fields. What are the connected components of this space? That is, when can one vector field be deformed into another along a path of horizontal vector fields? For simplicity, we only consider this in the case where the base orbifold $\Sigma$ is oriented. By proposition 4.1, the question reduces to studying homotopy classes of maps of the form

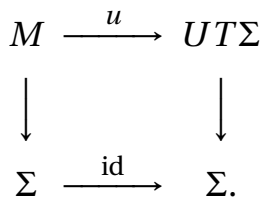

Consider two such maps $u, v: M \rightarrow U T \Sigma$ and suppose they have the same degree $d$. (Otherwise, they are clearly not homotopic.) By the averaging method used in the proof of proposition 4.4 we may assume there are metrics on the fibers of $M$ and $U T \Sigma$ and that $u$ and $v$ have the same constant speed on all fibers. As $\Sigma$ is oriented, the fibers of $U T \Sigma$ are oriented. Using the metric, there is then a well-defined difference map $u-v: M \rightarrow \mathbb{S}^{1}$ measuring the angle between vectors. This map is constant on fibers and therefore quotients to a map $g: \Sigma \rightarrow$ $\mathbb{S}^{1}$. The map $u$ is homotopic to $v$ if and only if $g$ is homotopic to a constant map. Since we are only concerned with the homotopy class of $g$, the smooth orbifold structure of $\Sigma$ is unimportant and we may consider $g$ as a map from $\Sigma_{0}$ to $\mathbb{S}^{1}$ where $\Sigma_{0}$ is the underlying topological surface.

Note that these steps are reversible. Given a continuous map $g: \Sigma_{0} \rightarrow \mathbb{S}^{1}$, we may, up to homotopy, assume $g$ is smooth everywhere and constant on a neighbourhood of the cone points. If $\pi: M \rightarrow \Sigma$ is the Seifert fibering, then $g \circ \pi$ : $M \rightarrow \mathbb{S}^{1}$ is a smooth map. Given $u$ as above, we may construct $v$ by $v=u+g \circ \pi$ where the plus sign denotes addition of angles. If we assume $u$ is fixed, then this gives a bijection between homotopy classes of maps $v: M \rightarrow U T \Sigma$ of the same degree as $u$ and homotopy classes of maps $g: \Sigma_{0} \rightarrow \mathbb{S}^{1}$.

There are canonical isomorphisms identifying

(1) the homotopy classes of maps from $\Sigma_{0}$ to $\mathbb{S}^{1}$,

(2) homomorphisms from $\pi_{1}\left(\Sigma_{0}\right)$ to $\mathbb{Z}=\pi_{1}\left(\mathbb{S}^{1}\right)$,

(3) homomorphisms from the first homology group $H_{1}\left(\Sigma_{0}\right)$ to $\mathbb{Z}$, and

(4) elements of the first cohomology group $H^{1}\left(\Sigma_{0}, \mathbb{Z}\right)$. 
For $\Sigma_{0}=\mathbb{S}^{2}$, this follows because all of the above are trivial. For other oriented surfaces, it follows because both $\Sigma_{0}$ and $\mathbb{S}^{1}$ are $K(\pi, 1)$. (See also Theorem 4.57 and the discussion on page 198 of [Hat02].)

Each connected component of the space of horizontal vector fields is therefore uniquely determined by the degree $d$ (which is also the number of turns that the vector field makes around any fiber) and an element of $H^{1}\left(\Sigma_{0}, \mathbb{Z}\right)$.

For a Seifert fibering $M \rightarrow \Sigma$ of an oriented manifold, let $\mathscr{D}(M \rightarrow \Sigma)$ denote the "allowable degrees" of a horizontal vector field. That is $d \in \mathscr{D}(M \rightarrow \Sigma)$ if and only if there is a degree- $d$ map $u: M \rightarrow U T \Sigma$ of the form given in proposition 4.1, The above reasoning may then be used to show the following.

Theorem 9.1. For a Seifert fibering $M \rightarrow \Sigma$ over an oriented orbifold, there is a bijection between the connected components of the space of horizontal vector fields and pairs of the form $(d, \varphi) \in \mathscr{D}(M \rightarrow \Sigma) \times H^{1}\left(\Sigma_{0}, \mathbb{Z}\right)$.

If $\chi(\Sigma) \neq 0$, then theorem 4.5 shows that the degree $d$ is uniquely determined by the fibering. In particular, for a bad, elliptic, or hyperbolic orbifold with $\Sigma_{0}=\mathbb{S}^{2}$, theorem 9.1 implies that the Seifert fibering uniquely determines the horizontal vector field up to homotopy. Conversely if $H^{1}\left(\Sigma_{0}, \mathbb{Z}\right)$ is non-trivial, then many homotopy classes are possible. For instance, consider the 3-torus $\mathbb{T}^{3}$ with fibers tangent to the vertical $z$-direction. Then $U T \mathbb{T}^{2}$ may be identified with $\mathbb{T}^{3}$ and the classes of horizontal vector fields correspond to maps of the form

$$
\mathbb{T}^{3} \rightarrow \mathbb{T}^{3}, \quad(x, y, z) \mapsto(x, y, a x+b y+d z)
$$

for all $(a, b, d) \in \mathbb{Z}^{3}$.

\section{MANIFOLDS WITH BOUNDARY}

In this final section, we consider Seifert fiberings on manifolds with boundary. First, consider the case where there are boundary conditions for the vector field. We could require that the vector field be either tangent or transverse to the boundary and the resulting restrictions on $M$ will be the same.

Theorem 10.1. For a Seifert fibering $M \rightarrow \Sigma$ on a manifold with boundary, the following are equivalent:

(1) M has a horizontal vector field everywhere tangent to the boundary,

(2) M has a horizontal vector field everywhere transverse to the boundary,

(3) the base orbifold $\Sigma$ is either the annulus or the Möbius band.

Proof. We show (2) $\Leftrightarrow$ (3). The proof of (1) $\Leftrightarrow$ (3) is similar and left to the reader. Note that proposition 4.4 holds for manifolds with boundary using the same proof. Suppose $M$ supports a horizontal vector field which is everywhere transverse to $\partial M$ and consider the associated map $u: M \rightarrow U T \Sigma$. For a point $x \in \partial \Sigma$, the restriction of $u$ to the fiber over $x$ cannot be surjective as its range omits the two unit vectors at $x$ which are tangent to $\partial \Sigma$. This implies that $u$ has degree zero 
and proposition 4.4 shows that $u$ is homotopic to a composition $M \rightarrow \Sigma \rightarrow U T \Sigma$. In the proof of the proposition, the vector field $\Sigma \rightarrow U T \Sigma$ is defined by averaging and one can verify that is it transverse to $\partial \Sigma$. Further, the same argument as in proposition 2.3 shows that $\Sigma$ has no cone points and is therefore a surface with boundary. The Poincaré-Hopf theorem then implies that $\Sigma$ is either the annulus or Möbius band.

Conversely, for any circle bundle over an annulus or Möbius band, we may compose the projection $M \rightarrow \Sigma$ with a vector field $\Sigma \rightarrow U T \Sigma$ transverse to $\partial \Sigma$ and produce a horizontal vector field on $M$ transverse to $\partial M$.

For the remainder of the section, we consider horizontal vector fields with no boundary conditions.

Theorem 10.2. Let $M \rightarrow \Sigma$ be a Seifert fibering on a manifold with boundary. Then $M$ has a horizontal vector field if and only if one or both of the following hold:

(1) the base orbifold $\Sigma$ is a surface with boundary, or

(2) $M$ fiberwise covers the unit tangent bundle of $\Sigma$.

To prove this, we use the following.

Lemma 10.3. An orbifold with boundary supports a non-zero vector field if and only if it has no cone points (i.e., it is a surface with boundary).

Proof. As shown in the proof of proposition 2.3, it is impossible to define a nonzero vector field in the neighbourhood of a cone point and so the existence of such a vector field implies that $\Sigma$ is a surface with boundary. Conversely, if we have no boundary conditions, we may construct a non-zero vector field on any surface with boundary. For instance, we may take a generic vector field on a closed surface. This is zero at finitely many points [Pei62], and we can excise one or more topological disks to remove all of these points.

Using this lemma and checking that the results of section 4 extend to the case of a manifold with boundary, one may then prove theorem 10.2

For an oriented manifold with boundary, write the Seifert invariant as

$$
\left(g, n ;\left(\alpha_{1}, \beta_{1}\right), \ldots,\left(\alpha_{k}, \beta_{k}\right)\right)
$$

where $n>0$ is the number of boundary components. We can reorder the pairs, add or remove pairs of the form $(1,0)$, and (specifically for $\partial M \neq \varnothing$ ) add an integer to any of the ratios $\beta_{i} / \alpha_{i}$ without changing the fibering. Note here that integer changes to one ratio $\beta_{i} / \alpha_{i}$ do not need to be offset with a change to another ratio $\beta_{j} / \alpha_{j}$. Because of this, the Euler number $e(M \rightarrow \Sigma)$ is not well defined. See $\$ 2$ of [Hat07] for more details.

By adapting arguments in section 4 one can prove the following analogue of theorem 4.5. Note that the condition $d \cdot e(M \rightarrow \Sigma)=\chi(\Sigma)$ has been removed. 
Proposition 10.4. Let $M$ be a manifold with boundary and let $M \rightarrow \Sigma$ be a Seifert fibering such that $\Sigma$ has one or more cone points. Then the following are equivalent:

(1) $M$ has a horizontal vector field;

(2) there is a continuous map $u: M \rightarrow U T \Sigma$ such that

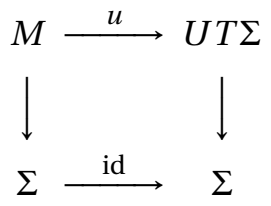

commutes;

(3) there is a fiberwise covering from $M$ to the unit tangent bundle UT $\Sigma$;

(4) $M$ is orientable, the fibering has Seifert invariant

$$
\left(g, n ;\left(\alpha_{1}, \beta_{1}\right), \ldots,\left(\alpha_{k}, \beta_{k}\right)\right)
$$

and there is a non-zero integerd such that

$$
d \beta_{i} / \alpha_{i} \equiv-1 / \alpha_{i} \quad \bmod \mathbb{Z}
$$

for all $i=1, \ldots, k$.

Even with the Euler number condition removed, it may not be possible to find a horizontal vector field. Consider, for instance $(g, n ;(3,1),(3,2))$. No integer $d$ satisfies $\frac{1}{3} d \equiv \frac{2}{3} d \equiv \frac{-1}{3} \bmod \mathbb{Z}$ and so no horizontal vector field exists.

If a Seifert fibering satisfies item (4) of proposition 10.4 for some integer $d$, then it also satisfies the condition when $d$ is replaced by $d+m \ell$ where $m$ is any integer and $\ell$ is the least common multiple of $\left\{\alpha_{1}, \ldots, \alpha_{k}\right\}$. Using this and adapting the results in section 9, one may show that if a Seifert fibering on a manifold with boundary has one horizontal vector field, then it has infinitely many homotopy classes of such vector fields.

Acknowledgements. The author thanks Jonathan Bowden, Heiko Dietrich, Rafael Potrie, Jessica Purcell, Mario Shannon, and Stephan Tillmann for helpful conversations. We also thank the reviewer for helpful comments. This research was partially funded by the Australian Research Council.

\section{REFERENCES}

[Bar96] Thierry Barbot. Flots d'Anosov sur les variétés graphées au sens de Waldhausen. Ann. Inst. Fourier (Grenoble), 46(5):1451-1517, 1996.

[Bro60] E. J. Brody. The topological classification of the lens spaces. Ann. of Math. (2), 71:163$184,1960$.

[Cho12] Suhyoung Choi. Geometric structures on 2-orbifolds: exploration of discrete symmetry, volume 27 of MSJ Memoirs. Mathematical Society of Japan, Tokyo, 2012.

[Con92] J. H. Conway. The orbifold notation for surface groups. In Groups, combinatorics \& geometry (Durham, 1990), volume 165 of London Math. Soc. Lecture Note Ser., pages 438447. Cambridge Univ. Press, Cambridge, 1992. 
[EHN81] David Eisenbud, Ulrich Hirsch, and Walter Neumann. Transverse foliations of Seifert bundles and self-homeomorphism of the circle. Comment. Math. Helv., 56(4):638-660, 1981.

[Ghy84] Étienne Ghys. Flots d'Anosov sur les 3-variétés fibrées en cercles. Ergodic Theory Dynam. Systems, 4(1):67-80, 1984.

[Hat02] Allen Hatcher. Algebraic topology. Cambridge University Press, Cambridge, 2002.

[Hat07] Allen Hatcher. Notes on basic 3-manifold topology, 2007.

[HPS18] Andy Hammerlindl, Rafael Potrie, and Mario Shannon. Seifert manifolds admitting partially hyperbolic diffeomorphisms. J. Mod. Dyn., 12:193-222, 2018.

[JN83] Mark Jankins and Walter D. Neumann. Lectures on Seifert manifolds, volume 2 of Brandeis Lecture Notes. Brandeis University, Waltham, MA, 1983.

[Nai94] Ramin Naimi. Foliations transverse to fibers of Seifert manifolds. Comment. Math. Helv., 69(1):155-162, 1994.

[Orl72] Peter Orlik. Seifert manifolds. Lecture Notes in Mathematics, Vol. 291. Springer-Verlag, Berlin-New York, 1972.

[Pei62] M. M. Peixoto. Structural stability on two-dimensional manifolds. Topology, 1:101-120, 1962.

[Sco83] Peter Scott. The geometries of 3-manifolds. Bull. London Math. Soc., 15(5):401-487, 1983.

School of Mathematical Sciences, Monash University, Victoria 3800 Australia

URL: http://users.monash.edu.au/ ahammerl/

E-mail address: andy. hammerlindl@monash.edu 